\title{
Global Non-Potential Magnetic Models of the Solar Corona During the March 2015 Eclipse
}

\author{
Anthony R. Yeates ${ }^{1}$ - Tahar Amari ${ }^{2}$ Ioannis Contopoulos ${ }^{3,4} \cdot$ Xueshang Feng $^{5}$. \\ Duncan H. Mackay ${ }^{6}$ - Zoran Mikić ${ }^{7}$. Thomas Wiegelmann ${ }^{8} \cdot$ Joseph Hutton $^{9}$. \\ Christopher A. Lowder ${ }^{10}$ • Huw Morgan ${ }^{9}$ Gordon Petrie ${ }^{11}$ - Laurel A. Rachmeler ${ }^{12}$. \\ Lisa A. Upton ${ }^{13}$ - Aurelien Canou ${ }^{2}$ - Pierre Chopin ${ }^{2}$ - Cooper Downs ${ }^{7}$. \\ Miloslav Druckmüller ${ }^{14}$ • Jon A. Linker ${ }^{7}$ Daniel B. Seaton ${ }^{15,16}$ • Tibor Török ${ }^{7}$
}

Received: 25 October 2017 / Accepted: 1 August 2018 / Published online: 8 August 2018

(C) The Author(s) 2018

\begin{abstract}
Seven different models are applied to the same problem of simulating the Sun's coronal magnetic field during the solar eclipse on 2015 March 20. All of the models are non-potential, allowing for free magnetic energy, but the associated electric currents are developed in significantly different ways. This is not a direct comparison of the coronal modelling techniques, in that the different models also use different photospheric boundary conditions, reflecting the range of approaches currently used in the community. Despite the
\end{abstract}

$\triangle$ A.R. Yeates

anthony.yeates@durham.ac.uk

1 Department of Mathematical Sciences, Durham University, Science Laboratories, South Road, Durham, DH1 3LE, UK

2 CNRS, Centre de Physique Théorique de l'Ecole Polytechnique, 91128 Palaiseau Cedex, France

3 Research Center for Astronomy and Applied Mathematics, Academy of Athens, 11527 Athens, Greece

4 National Research Nuclear University (MEPhI), Moscow 115409, Russia

5 State Key Laboratory of Space Weather, National Space Science Center, Chinese Academy of Sciences, Beijing 100190, China

6 School of Mathematics and Statistics, University of St Andrews, North Haugh, St Andrews, Fife, KY16 9SS, UK

7 Predictive Science, Inc., 9990 Mesa Rim Rd., Ste. 170, San Diego, CA 92121-2910, USA

8 Max-Planck Institut für Sonnensystemforschung, Justus-von-Liebig-Weg 3, 37077 Göttingen, Germany

9 Institute of Mathematics, Physics \& Computer Sciences, Aberystwyth University, Penglais, Aberystwyth, Ceredigion, SY23 3BZ, UK

10 Southwest Research Institute, 1050 Walnut Street, Boulder, CO 80302, USA

11 National Solar Observatory, Boulder, CO 80303, USA

12 NASA Marshall Space Flight Center, Huntsville, AL 35811, USA

13 High Altitude Observatory, National Center for Atmospheric Research, 3080 Center Green Dr., Boulder, CO 80301, USA 
significant differences, the results show broad agreement in the overall magnetic topology. Among those models with significant volume currents in much of the corona, there is general agreement that the ratio of total to potential magnetic energy should be approximately 1.4. However, there are significant differences in the electric current distributions; while static extrapolations are best able to reproduce active regions, they are unable to recover sheared magnetic fields in filament channels using currently available vector magnetogram data. By contrast, time-evolving simulations can recover the filament channel fields at the expense of not matching the observed vector magnetic fields within active regions. We suggest that, at present, the best approach may be a hybrid model using static extrapolations but with additional energization informed by simplified evolution models. This is demonstrated by one of the models.

Keywords Magnetic fields $\cdot$ Sun: surface magnetism $\cdot$ Sun: corona

\section{Introduction}

In recent years, a number of different approaches have been developed for modelling nonpotential magnetic fields in the Sun's atmosphere, based on measurements of the magnetic field on the solar surface. Non-potential means that electric currents are allowed to be present within the coronal volume, in contrast to traditional potential-field extrapolations. However, different modellers use not only different approximations for the coronal magnetic field but also different input data and boundary conditions. To date, a direct comparison of these various approaches has been lacking in the literature. With this in mind, we convened a scientific team at the International Space Science Institute in Bern, Switzerland, and we report here on the results.

In this paper, we present a number of non-potential models side-by-side in a way that enables a direct comparison. Since the problem of coronal modelling is very much an active area of research, there are inevitable disagreements between the models. By highlighting the similarities and differences between different modelling approaches, and why they occur, we hope to assist the community in moving toward a single non-potential modelling approach for the corona. We would like to highlight what factors should be taken into account, and what are the biggest uncertainties in existing models.

We have fixed a single date and time, coinciding with the total solar eclipse of 2015 March 20. Fixing a single time allows us to compare both static and time-dependent models, and we chose an eclipse date during the Solar Dynamics Observatory (SDO) era so as to maximize the available observations of the real coronal structure. Figure 1 shows the structure of the observed corona on that day. There are no large active regions on the visible solar disk, although there is some activity on the far side and visible above both East and West limbs. The streamer structure is relatively complex, consistent with the fact that this eclipse occurred shortly after Solar Maximum. The streamers also show an asymmetry between the north and south poles, consistent with the formation of a polar coronal hole in the south but not in the north (Petrie 2017). In terms of non-potential structure, there is a clear polar

14 Faculty of Mechanical Engineering, Brno University of Technology, 61669 Brno, Czech Republic

15 Cooperative Institute for Research in Environmental Sciences, University of Colorado, Boulder, CO 80305, USA

16 National Centers for Environmental Information, National Oceanic and Atmospheric Administration, Boulder, CO 80305, USA 


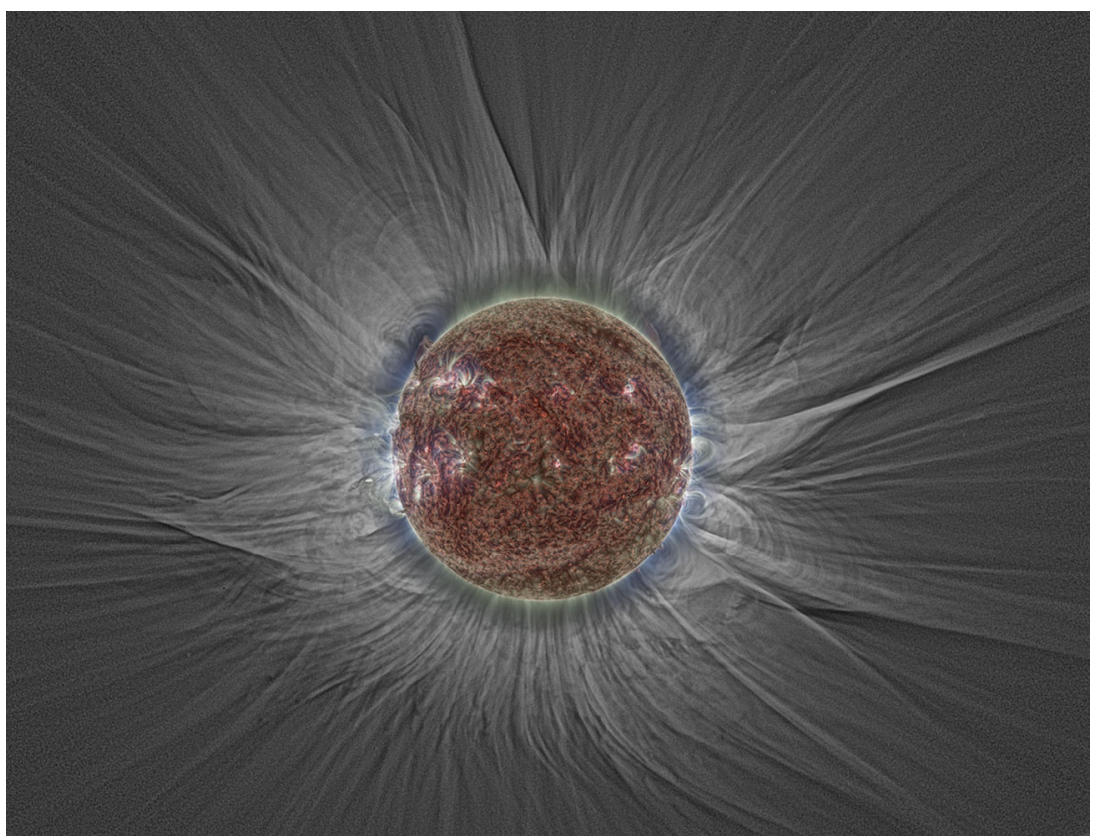

Fig. 1 An extreme-ultraviolet and white-light composite of the corona on 2015 March 20. The white light image is a combination of 29 exposures made from Longyearbyen, Svalbard, and aligned with sub-pixel precision using the Phase Correlation technique (Druckmüller 2009). This is overlaid with a combination of 171, 193 and $211 \AA$ (red, green, blue respectively) channels from the AIA instrument on the Solar Dynamics Observatory satellite. Each of the channels were processed using Multiscale Gaussian Normalization (MGN, Morgan and Druckmüller 2014) prior to combination

crown prominence on the north-east limb, with suggestions of a coronal cavity surrounding it. Although this is not a time of particularly high solar activity, the coronal structure is nevertheless more complex than that typically found around Solar Minimum. It is therefore quite challenging to model all of the aspects of this observed corona within the context of a single global magnetic field model.

The compared models are summarized in Sect. 2. All cover the full global solar corona, except for the polar regions in some cases. The outer boundaries of the models vary; for the comparisons in this paper we will therefore focus on the region $R_{\odot} \leq r \leq 2.5 R_{\odot}$ that is covered by all of the models. Grid resolution and boundary conditions were chosen by each individual modeller. In particular, different photospheric boundary conditions were used for each model, and these differences in input data are highlighted in Sect. 2. All models used magnetic data from the same SDO/HMI instrument, but different methods were used to reconstruct boundary maps of the full solar surface, leading to quite different photospheric magnetic fields. Owing to these differences in input data, our study should not be viewed as a direct side-by-side comparison of numerical codes for modelling the coronal magnetic field, such as (for example) the active region exercises by Schrijver et al. (2008) and DeRosa et al. (2015). Rather, the approach is more qualitative: to compare and contrast the results from different non-potential modelling approaches, where these approaches include the different methods of processing magnetogram input data that are characteristic of present-day coronal modelling. Through this we can assess the strengths and weaknesses of each approach. 
Because we only observe (usually) one side of the Sun, it is difficult to make detailed comparisons with observations using only a single snapshot such as this. Moreover, the lack of direct magnetic measurements above the photosphere is a major driver for the development of these models in the first place. Nevertheless, we are able to compare with indirect observations of the coronal magnetic field, and do so qualitatively in this paper. It is hoped that by combining all available information, we can ultimately build a "best guess" picture of the coronal magnetic structure during the eclipse. Following our summary of the models in Sect. 2, the resulting coronal magnetic fields are compared in Sect. 3, both with each other and with the indirect observations. The overall findings are discussed in Sect. 4.

\section{Non-Potential Models}

The models presented in this paper are summarized in Table 1, and their computational domains are summarized in Table 2. The latter table also shows magnetic energies, which will be discussed in Sect. 3. For purposes of illustration we have selected a single representative model of each type, although additional runs were carried out in many cases. These models are still under development, and not yet freely available, but readers interested in their use are encouraged to contact the individual modellers, as listed in Table 1.

Space precludes a detailed description of each model, so the following subsections concentrate on their similarities and differences. For a review of the various non-potential modelling approaches, see Mackay and Yeates (2012) or Wiegelmann et al. (2017), or follow the references given in Table 1. Some additional details are given for the $m h d$-mas and $m f$ models in Appendices A and B respectively, since these differ somewhat in their setup from previously published simulations.

We stress that, although we consider only a single model of each type, it would be possible to "mix and match" aspects of different models in future. For example, the techniques used to energize filament channels in the mhd-mas model could equally be applied to any

Table 1 Summary of the non-potential models presented

\begin{tabular}{|c|c|c|}
\hline Model & Input Data & Contact \\
\hline $\begin{array}{l}\text { nlf-op - Optimization NLFFF } \\
\text { Wiegelmann (2007), Tadesse et al. (2014) }\end{array}$ & HMI synoptic B & TW \\
\hline $\begin{array}{l}n l f-g r \text { - Grad-Rubin NLFFF } \\
\text { Amari et al. }(2013,2014)-\text { XTRAPOLS }\end{array}$ & HMI synoptic B + HARP & $\mathrm{TA}$ \\
\hline $\begin{array}{l}m h s-\text { Linear MHS } \\
\text { Bogdan and Low (1986) }\end{array}$ & HMI synoptic $B_{r}$ & TW \\
\hline $\begin{array}{l}f f e-\text { Force-free electrodynamics } \\
\text { Contopoulos et al. (2011), Contopoulos (2013) }\end{array}$ & HMI synoptic B & IC \\
\hline $\begin{array}{l}m f-\text { Evolving magnetofrictional } \\
\text { Mackay and van Ballegooijen (2006), Yeates (2014) }\end{array}$ & $B_{r}$ from HMI-driven $\mathrm{AFT}^{\mathrm{a}}$ & DHM \\
\hline $\begin{array}{l}\text { mhd-cese-MHD } \\
\text { Feng et al. (2012) }\end{array}$ & HMI synoptic $B_{r}$ & $\mathrm{XF}$ \\
\hline $\begin{array}{l}\text { mhd-mas - Zero-beta MHD } \\
\text { Mikic and Linker (1994), Mikić et al. (1999) }\end{array}$ & HMI synoptic $B_{r}+$ channels $^{\mathrm{b}}$ & $\mathrm{ZM}$ \\
\hline
\end{tabular}

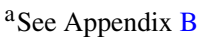

${ }^{\mathrm{b}}$ Filament channel locations based on $m f$ model (see Appendix A)
} 
Table 2 Domains, resolutions and energies ${ }^{\mathrm{a}}$ of the non-potential models

\begin{tabular}{llllll}
\hline Model & Boundaries $(\theta, r)$ & Resolution $(r, \theta, \phi)$ & $E\left[10^{33} \mathrm{ergs}\right]$ & $E_{\mathrm{p}}\left[10^{33} \mathrm{ergs}\right]$ & $E / E_{\mathrm{p}}$ \\
\hline nlf-op & $\pm 70^{\circ}, 2.56 R_{\odot}$ & $180 \times 280 \times 720$ & 3.24 & 2.32 & 1.40 \\
nlf-gr & $\pm 90^{\circ}, 2.5 R_{\odot}$ & $208 \times 250 \times 500$ & 3.47 & 3.42 & 1.01 \\
mhs & $\pm 70^{\circ}, 2.56 R_{\odot}$ & $180 \times 280 \times 720$ & 3.30 & 2.32 & 1.42 \\
ffe & $\pm 90^{\circ}, 5.8 R_{\odot}$ & $125 \times 225 \times 450$ & 3.78 & 3.50 & 1.79 \\
mf & $\pm 89^{\circ}, 2.5 R_{\odot}$ & $56 \times 180 \times 360$ & 2.63 & 1.65 & 1.47 \\
mhd-cese & $\pm 90^{\circ}, 30.0 R_{\odot}$ & $61^{\mathrm{b}} \times 92 \times 182$ & 1.99 & 1.72 & 1.21 \\
mhd-mas & $\pm 90^{\circ}, 2.5 R_{\odot}$ & $182 \times 200 \times 560$ & 2.52 & 1.47 \\
\hline
\end{tabular}

${ }^{\mathrm{a}}$ Energies are computed between $r=R_{\odot}$ and $r=2.5 R_{\odot}$

${ }^{\mathrm{b}}$ The resolution of 61 points in $r$ refers to the region $r \leq 2.5 R_{\odot}$ only

of the other static models. Or thermodynamics effects could be included in the mhd-mas model. Thus our comparison should be treated only as a representative selection, aiming to illustrate the consequences of including different model features.

\subsection{Modelling Approaches}

In the low corona, on a global scale, magnetic forces dominate over gravity and pressure gradients. Thus the large-scale magnetic field configuration is expected to be approximately force-free at the heights $r<2.5 R_{\odot}$ of interest here (Wiegelmann and Sakurai 2012). This means that the Lorentz force vanishes and the magnetic field $\mathbf{B}$ satisfies

$$
\mathbf{j} \times \mathbf{B}=0, \quad \nabla \cdot \mathbf{B}=0,
$$

where $\mathbf{j}=c \nabla \times \mathbf{B} /(4 \pi)$ is the electric current density. In a non-potential magnetic field with free energy, we must have $\mathbf{j} \neq 0$ at least in some regions of the corona. All of the models in Table 1 aim to approximate force-free equilibria of the form (1), except for $m h s$ and $m h d$ cese where plasma pressure and gravity also influence the equilibria. Namely, the $m h s$ model uses a special class of magnetohydrostatic equilibria where the electric current density flows on spherical shells. Here we set the parameter $a=1.0$ (for more details and the definition of $a$, see Bogdan and Low 1986). In this model, the radial magnetic field $B_{r}$ on $r=R_{\odot}$ is used as a boundary condition for spherical harmonic decomposition. The resulting magnetic field contains a non-vanishing Lorentz force, which is compensated by pressure gradients and gravity. The density and plasma pressure distribution are computed as a superposition of these terms and a stratified solar atmosphere model. The mhd-cese model solves the full magnetohydrodynamic (MHD) equations including gravity, centrifugal and Coriolis terms, as well as a volumetric heating term, relaxing to a static equilibrium that extends out to $r=30 R_{\odot}$, albeit at a lower resolution than the other models. All of the other models have an outer boundary at or near $r=2.5 R_{\odot}$, except for ffe where the computational domain extends to $5.8 R_{\odot}$, although a perfectly-matched (absorbing) layer is implemented above $2.5 R_{\odot}$.

Whilst the nlf-op, nlf-gr, ffe, $m f$ and mhd-mas models all use some form of iteration toward $\mathbf{j} \times \mathbf{B}=0$ - constrained by the imposed photospheric boundary conditions - they differ significantly in how the electric current structures in the final magnetic field are built up. In the first three models, the electric current distribution is fixed by the photospheric 
boundary conditions. By contrast, in the $m f$ model the coronal magnetic field is driven by large-scale horizontal flows in the photosphere, at the same time as relaxing the coronal magnetic field toward $\mathbf{j} \times \mathbf{B}=0$. This leads to an evolving photospheric $B_{r}$ distribution. The advantage of this approach is that it can build up large-scale current structures in a manner mimicking that in the real Sun. But the disadvantage is that a whole time series of input data extending over several months is required on the photospheric surface, both for $B_{r}$ and the horizontal flows. Moreover, the final $B_{r}$ distribution in the $m f$ model matches that of the original magnetogram only on a coarse scale. The mhd-mas model is in some sense a hybrid: although it uses a single observed map of $B_{r}$ on the solar surface, additional electric fields are imposed to drive the formation of filament channels at specified locations in the corona. The locations of these channels, and in particular their chirality (direction of the transverse magnetic field) were guided by the results of the $m f$ model and by EUV observations (see Appendix A).

\subsection{Photospheric Boundary Conditions}

The different modelling techniques each require particular input data at the solar photosphere. All of the models except $m f$ use only a single magnetic map covering the full photospheric surface, notionally at the time of the eclipse. Vector magnetic data with all three components of $\mathbf{B}$ are required for $n l f-o p, n l f-g r$ and ffe, whereas only the radial component $B_{r}$ is utilized by all of the other models.

Since the observed vector magnetograms are taken at photospheric heights where the magnetic field is not generally force free, these are generally not consistent with (1). The three models that use vector magnetic input deal with this in different ways. The $n l f$ - $g r$ model uses the Grad-Rubin scheme. By imposing the force-free function $\alpha=j_{r} / B_{r}$ in only one polarity (here, $B_{r}>0$ ), as well as $B_{r}$ everywhere, it matches the transverse components of B in locations of this polarity. Some cut-offs on the $B_{r}$ and on the transverse components of $\mathbf{B}$ are applied so as to generate regular values of $\alpha$, and in particular it is assumed that $j_{r}=0$ in regions of weak $B_{r}$. The $n l f$-op method uses an optimization that penalizes differences in the simulated and observed vector $\mathbf{B}$ over the whole map, but allows differences, particularly in pixels with smaller observed transverse field. The ffe method imposes the vector $\mathbf{B}$ over the whole map, although this leads to a boundary layer just above the photosphere where $\mathbf{B}$ becomes more force free.

The $m h s, m f$, $m h d$-cese and $m h d$-mas models require only the observed radial magnetic field $B_{r}$ on the photospheric boundary rather than the full vector $\mathbf{B}$, although as mentioned above the $m f$ model requires a whole time sequence of $B_{r}$ measurements. In the BogdanLow solution used by the mhs model, the transverse components of $\mathbf{B}$ on the boundary are uniquely determined by the form of the solution. However, in the methods used by $m f, m h d$ cese and mhd-mas, a transverse electric field is required to specify a unique evolution from the initial potential field.

This transverse electric field is set either by boundary flows or through ad-hoc relations. In $m h d$-cese, the transverse electric field is simply set to zero as the corona is evolved toward equilibrium (the initial plasma parameters are taken from Parker's hydrodynamic isothermal solar wind solution). In mhd-mas, the transverse electric field is set to zero except where it is imposed to create magnetic flux ropes in filament channels (see Appendix A). The $m f$ model is rather different since transverse electric fields are imposed continually throughout the 200-day evolution, so as to evolve the photospheric $B_{r}$ distribution as well as the coronal magnetic field. This is described in more detail in Appendix B. 


\subsection{Magnetogram Input Data}

All models used photospheric magnetogram data from the HMI instrument on Solar Dynamics Observatory (Schou et al. 2012). However, it is important to note that these data were processed in different ways by each modeller, leading to different distributions of $B_{r}$ on $r=R_{\odot}$. Rather than standardize this processing and do a direct model comparison, we have decided to highlight the fact that the input data themselves represent a significant difference between present models of the coronal magnetic field. The differences are summarized in this section.

Several of the models used a custom SDO/HMI vector map that was created specially for this project, shown in Fig. 2. Here data from the successive Carrington rotations CR2161 and CR2162 were combined so as to extract a synoptic map centered on the eclipse Central Meridian (Carrington longitude $\approx 99^{\circ}$ during CR2161), with the discontinuity located $180^{\circ}$ out of phase from this (at Carrington longitude $279^{\circ}$ ). The data were processed using the standard HMI pipeline (Hoeksema et al. 2014), but with azimuth-angle disambiguated fulldisk images. Strong fields $(|\mathbf{B}| \geq 50 \mathrm{G})$ were left unsmoothed, but boxcar smoothing was applied to low-latitude quiet-Sun fields $(|\mathbf{B}|<50 \mathrm{G})$, in order to improve the signal-to-noise ratio. High latitude fields (poleward of $\pm 70^{\circ}$ latitude) were filled using a combination of smoothed vector $B_{r}$ and radially-corrected line-of-sight field.

For the ffe model, this custom vector map (with dimensions $1440 \times 3600$ ) was simply boxcar binned to a lower resolution. To generate the initial potential fields for the nlf-op and $m h s$ models, the map was initially smoothed by retaining only spherical harmonics of degree $\ell \leq 25$. The full map was then used in the ensuing iteration for $n l f-o p$, while the $m h s$ input map remains smoothed. For $n l f$-op, note that no pre-processing of the vector data (as described by Tadesse et al. 2009) was applied.

The $n l f$-gr model took the basic map as a starting point but made two modifications. Firstly, $B_{r}$ on the visible disk (centred at Carrington longitude $99^{\circ}$ ) was replaced by an

Fig. 2 The $B_{r}, B_{\theta}$, and $B_{\phi}$ components of the custom $\mathrm{SDO} / \mathrm{HMI}$ vector map, with color tables saturated at $\pm 50 \mathrm{G}$ (red positive, blue negative). The horizontal axis shows Carrington longitude and the vertical dashed line marks the location of central meridian at the time of eclipse $\left(99^{\circ}\right)$. The vertical solid line marks the time discontinuity between data from the synoptic maps for CR2161 (to the left) and CR2162 (to the right). This is at $279^{\circ}$ rather than the typical $360^{\circ}$

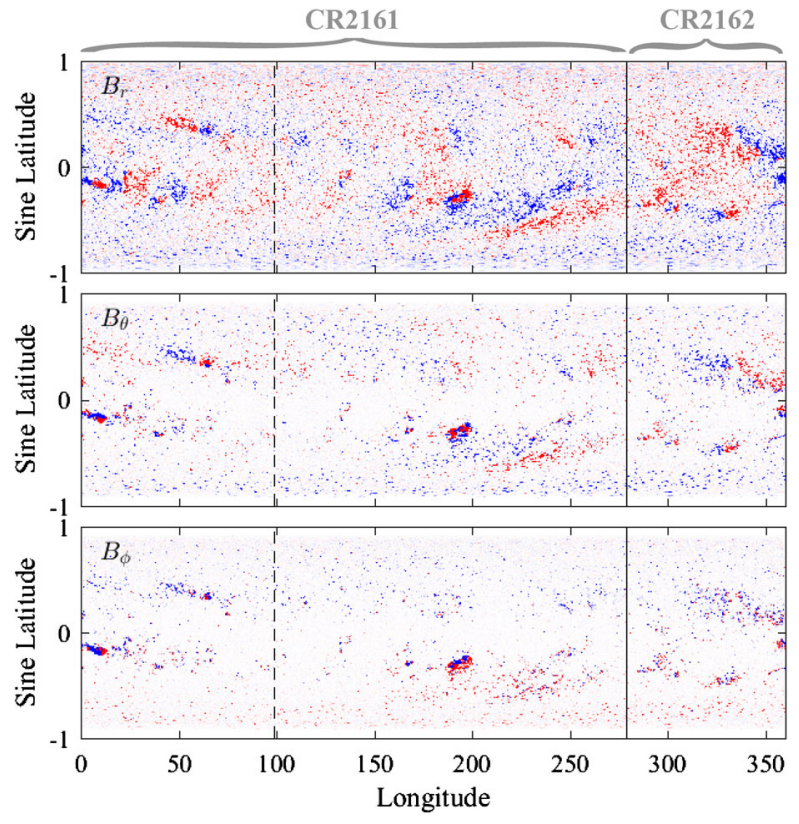


HMI longitudinal 720-second full-disk magnetogram from 09:47:58 UT on 2015 March 20. Secondly, six active regions on the visible disk were replaced by HMI vector Space-Weather HMI Active Region Patches (HARPs; Bobra et al. 2014). Specifically, patches number 5337, $5342,5345,5347,5348$, and 5350, again observed at 09:47:58 UT. These additions were made on a higher resolution grid $(3804 \times 5784)$, which was subsequently interpolated to a final mesh of $250 \times 500$ for the computation. This final mesh is non-uniform with more grid points within active regions.

The mhd-cese model used a standard SDO/HMI synoptic magnetogram for CR2161, smoothed with a simple Gaussian kernel (as a function of heliocentric angle). The mhd-mas model used a $B_{r}$ map constructed from HMI data in a similar way to the custom map, combining synoptic maps from CR2161 (from $0^{\circ}$ to $279^{\circ}$ ) and CR2162 (from $279^{\circ}$ to $360^{\circ}$ ). The high-latitude fields were filled in from previous Carrington rotations when the poles were visible, and the maps were suitably smoothed for the MHD calculation. The $m f$ model did not use HMI data directly, being driven instead by a flux transport model coupled with the emergence of bipolar magnetic regions starting from 2014 September 1 - this is described in Appendix B.

It is important to note that the models using synoptic input data from both CR2161 and CR2162 do not represent what could have been achieved in an advance prediction of the eclipse, since the maps incorporate magnetogram data taken after the eclipse time. The exceptions to this are $m f$ and $m h d$-cese, which use only information from the visible face of the Sun up to but not after the eclipse.

Figure 3 shows the distribution of radial magnetic field at the photosphere $r=R_{\odot}$ in each model (at the closest simulated time to the eclipse for the $m f$ model). It is clear that there are differences between these input data for the different models. Most obvious are the variations in resolution and in the amount of smoothing applied to the data, as described above. But important also are the timing variations, namely how recently different regions of the maps have been updated. There are two notable differences that affect the model results presented below. Firstly, a small active region (NOAA 12304) is located at approximately $140^{\circ}$ Carrington longitude, in the Northern hemisphere. This region is visible in the EUV eclipse image (Fig. 1), in the Northern hemisphere between disk center and the West limb. However, it is missing from the HMI vector synoptic map in Fig. 2 because it emerged after that Carrington longitude had rotated past Central Meridian. Accordingly, it is omitted from models $n l f$-op, mhs, ffe, mhd-cese and mhd-mas. It is included, however, in $n l f-g r$ thanks to the replacement of the visible disk by an HMI full-disk magnetogram taken just prior to the eclipse. The region is also included in the $m f$ model owing to the method of bipolar magnetic region (BMR) insertion (Appendix B).

The second difference is potentially more significant: all models except for $m f$ and $m h d$ cese include the activity complex centred around $10^{\circ}$ Carrington longitude in the Southern hemisphere, and appearing at the East limb in Fig. 1. In the $m f$ model the corresponding BMRs have not yet been assimilated at the time of eclipse, since they were not yet fully visible at that time and their properties could not be determined. Similarly they do not appear in mhd-cese since the corresponding longitudes were taken from earlier in CR2161, rather than CR2162 as in the other models. Such differences are typical of coronal magnetic field modelling based on synoptic magnetic field observations, but must be taken into account when assessing our results, which we consider next. We reiterate that the inclusion of this activity complex in the other models was possible only by using observations taken after the eclipse; if the comparison had been carried out in real time then this correction would not have been possible. 


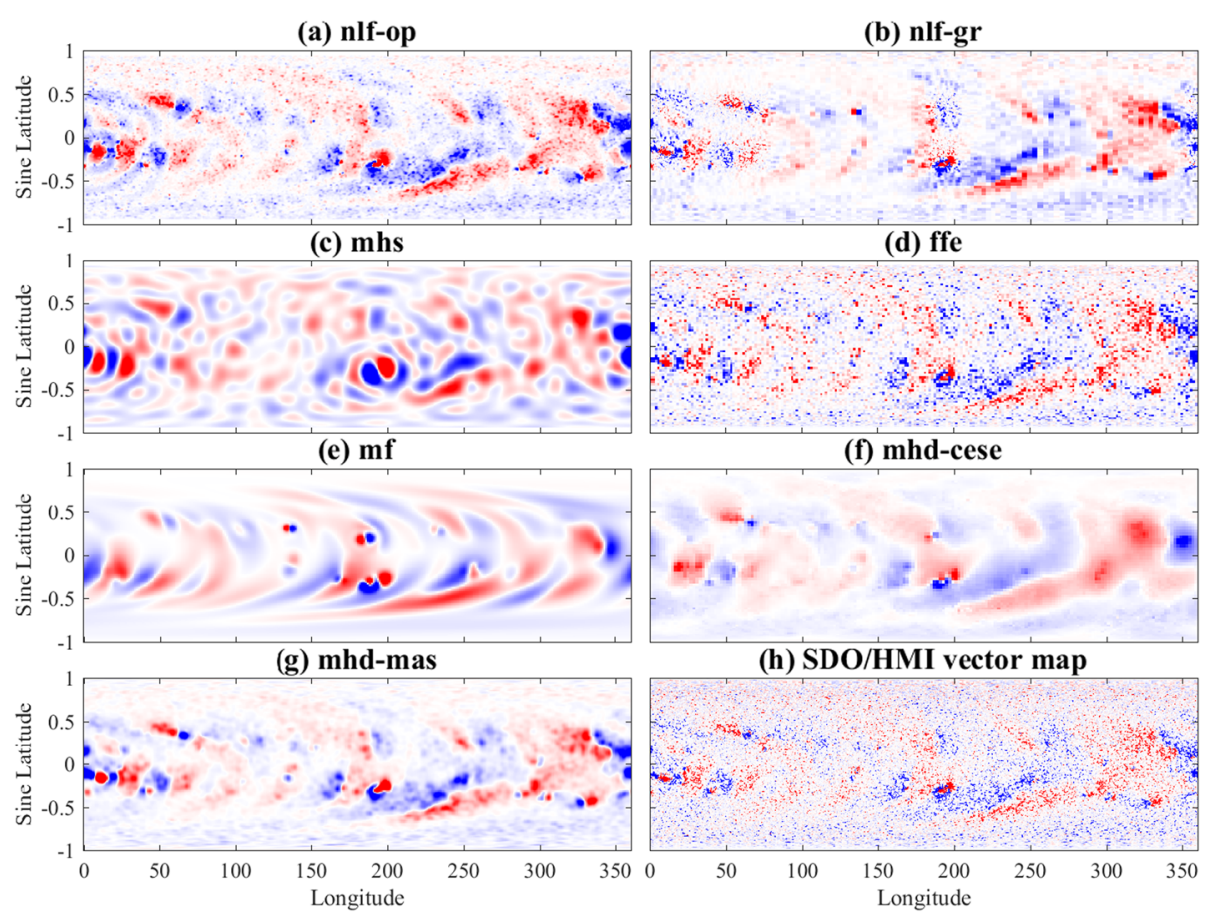

Fig. 3 Radial magnetic field $B_{r}\left(R_{\odot}, \theta, \phi\right)$ in each model, over the full solar surface. Here the horizontal axis shows Carrington longitude, with Central Meridian at eclipse time located at $99^{\circ}$. The color tables are saturated at $\pm 50 \mathrm{G}$ (red positive, blue negative). Panel (h) repeats $B_{r}$ from the SDO/HMI map in Figure 2, for ease of comparison

\section{Model Comparison}

A number of aspects of the models are compared in the following subsections, with the aim of highlighting the similarities and differences between them. Since many of the models do not include realistic thermodynamics (or indeed any plasma at all), we limit our comparison to the magnetic field, rather than plasma density or temperature. Some of the models, such as the mhd-mas model, have already been developed extensively to describe the flow of energy in the corona, as well as the acceleration of the solar wind, and can deduce the coronal temperature and density. In fact, we also studied this eclipse using this more sophisticated version of the mhd-mas model, but we do not describe those results here since they exceed the scope of the comparisons presented in this paper. Ground truth observations of the real corona are included, although these can provide only indirect, qualitative information about the coronal magnetic field.

\subsection{Magnetic Flux and Energy}

We begin with some overall diagnostics. Firstly, Fig. 4(a) shows the total unsigned magnetic flux,

$$
\Phi(r)=\int_{0}^{2 \pi} \int_{\theta_{\min }}^{\theta_{\max }}\left|B_{r}(r, \theta, \phi)\right| r^{2} \sin \theta \mathrm{d} \theta \mathrm{d} \phi,
$$



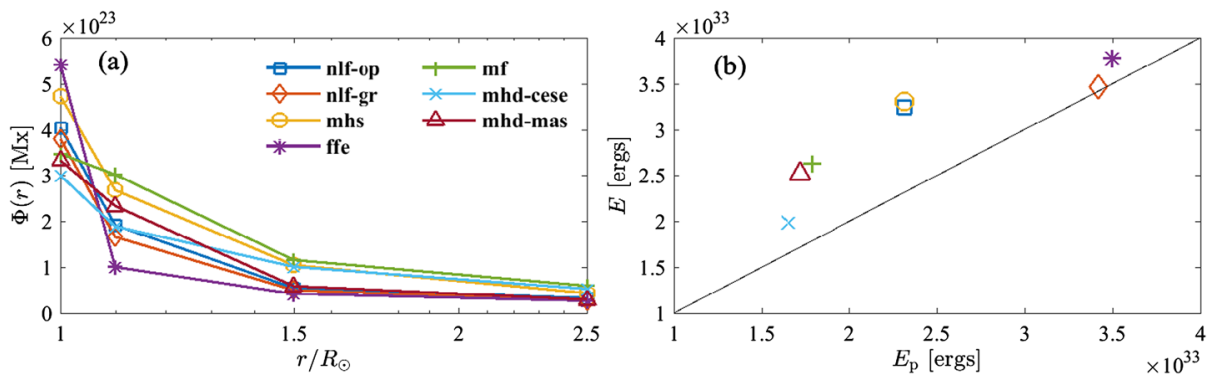

Fig. 4 Magnetic flux (a) and energy (b) of the models. Shown are (a) unsigned radial magnetic flux $\Phi(r)$ as a function of height, and (b) total magnetic energy $E$ versus potential magnetic energy $E_{\mathrm{p}}$. The solid line indicates $E=E_{\mathrm{p}}$

for each model at a sequence of heights in the corona. At $r=R_{\odot}$ there are significant differences in the photospheric flux arising from the difference in resolution between the models (these different resolutions are stated in Table 2 and evident in Fig. 3). The model photospheric fluxes span the range $3-5.5 \times 10^{23} \mathrm{Mx}$, from $m$ hd-cese with the lowest flux to ffe with the highest. For comparison, note that the SDO/HMI synoptic map in Fig. 2 has a flux $\Phi\left(R_{\odot}\right)=5.8 \times 10^{23} \mathrm{Mx}$ at its original resolution. The difference in resolution between the models becomes insignificant above $1.5 R_{\odot}$, where the behaviour is dominated by low-order spherical harmonics. Accordingly, most of the models predict a similar open magnetic flux of around $3 \times 10^{22} \mathrm{Mx}$. The exceptions are $m h s, m f$ and $m h d$-cese, where the open flux is inflated due to the presence of significant volumetric currents in the corona up to $6 \times 10^{22} \mathrm{Mx}$ in the case of $m f$. The $m f$ model includes the ejection of magnetic flux ropes which also increases the open flux. These open flux values, and the open magnetic field distribution, will be further discussed in Sect. 3.5.

Figure 4(b) shows the total magnetic energy of each model between $r=R_{\odot}$ and $r=$ $2.5 R_{\odot}$, given by

$$
E=\int_{R_{\odot}}^{2.5 R_{\odot}} \int_{0}^{2 \pi} \int_{\theta_{\min }}^{\theta_{\max }} \frac{|\mathbf{B}(r, \theta, \phi)|^{2}}{8 \pi} r^{2} \sin \theta \mathrm{d} \theta \mathrm{d} \phi \mathrm{d} r,
$$

and plotted against the corresponding energy $E_{\mathrm{p}}$ for a potential field source surface extrapolation with source surface at $r=2.5 R_{\odot}$ and the same $B_{r}\left(R_{\odot}, \theta, \phi\right)$ for each particular model. These energies are also listed in Table 2. There are considerable variations in both $E$ and $E_{\mathrm{p}}$, which depend to some extent on the model resolution. The distance of each symbol above the solid line indicates the excess of non-potential energy $E$ above $E_{\mathrm{p}}$, or the relative free energy. This varies from very little free energy for $n l f$ - $g r$ to around $40 \%$ for $n l f$-op, $m h s$, $m f$ and $m h d$-mas. Whilst the latter models all produce a similar percentage of free energy, the absolute values differ significantly, and we will see in Sect. 3.2 that the distributions of electric current within the volume also differ significantly. In the case of the mhs model, the free energy depends on the choice of the $a$ parameter; for example, taking $a=2$ would lead to $E=1.73 E_{\mathrm{p}}$ rather than $1.42 E_{\mathrm{p}}$ for $a=1$. The particularly low free energy for $n l f$ - $g r$ arises because the electric currents are strongly concentrated within active regions, with the magnetic field being close to potential throughout most of the volume. 
Fig. 5 Measures of the (a) perpendicular and (b) parallel currents for each model, as functions of height. Panel (c) shows the ratio of parallel to perpendicular measures
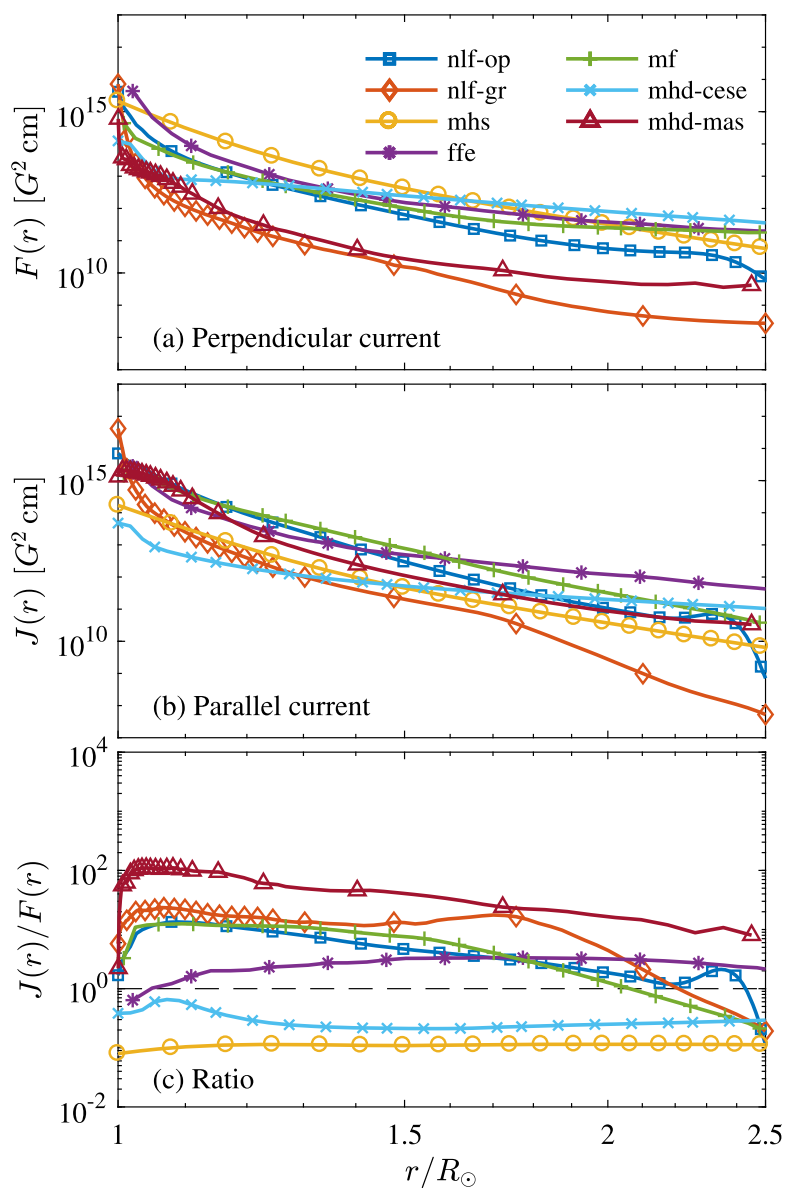

\subsection{Electric Currents}

Figures 5(a) and (b) show measures of the average perpendicular and parallel electric currents in each model, as a function of radius. Specifically we plot the quantities

$$
F(r)=\int_{0}^{2 \pi} \int_{\theta_{\min }}^{\theta_{\max }}|(\nabla \times \mathbf{B}) \times \mathbf{B}| r^{2} \sin \theta \mathrm{d} \theta \mathrm{d} \phi
$$

and

$$
J(r)=\int_{0}^{2 \pi} \int_{\theta_{\min }}^{\theta_{\max }}|(\nabla \times \mathbf{B}) \cdot \mathbf{B}| r^{2} \sin \theta \mathrm{d} \theta \mathrm{d} \phi .
$$

The perpendicular measure $F(r)$ is essentially a measure of the Lorentz force $\mathbf{j} \times \mathbf{B}$. Figure 5(c) shows the ratio $J(r) / F(r)$, which is a measure of "force-freeness" for each model.

Below about $r=1.5 R_{\odot}$, the ratio $J / F$ divides the models into three broad classes: those models that are relatively force-free with $J / F \gg 1$ (mhd-mas, $n l f$-gr, $n l f-o p$ and $m f$ ), those that are not force-free, with $J / F<1$ (mhd-cese and $m h s$ ), and the ffe model which is not 


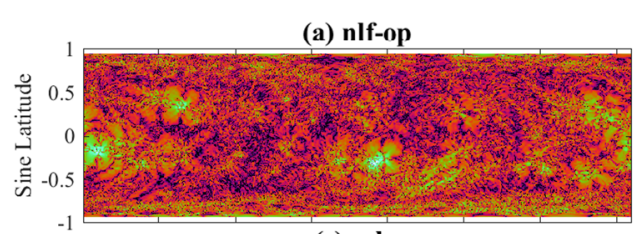

(c) $\mathrm{mhs}$

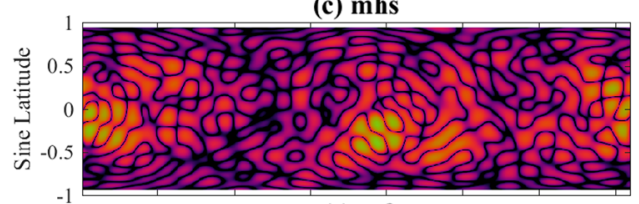

(e) $\mathrm{mf}$

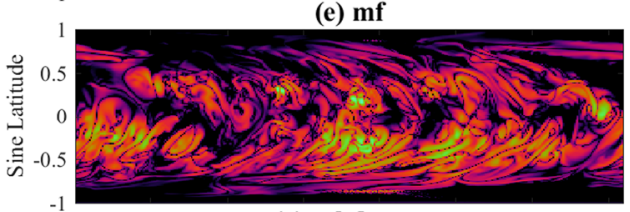

(g) mhd-mas

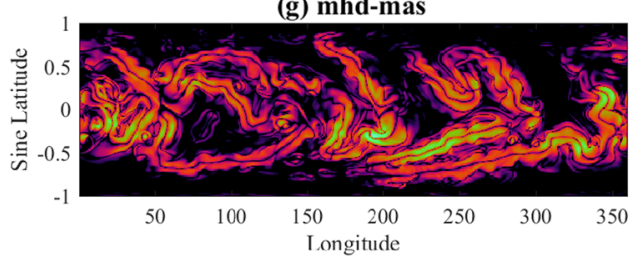

(b) nIf-gr

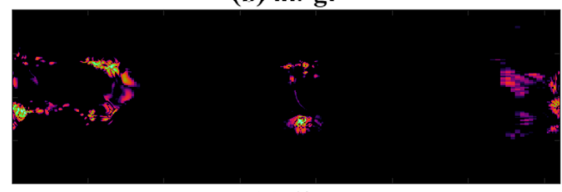

(d) ffe

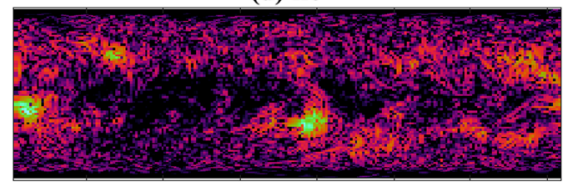

(f) mhd-cese

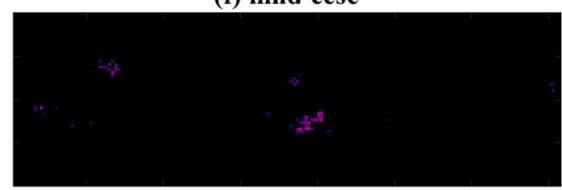

(h) SDO/HMI map (photosphere)

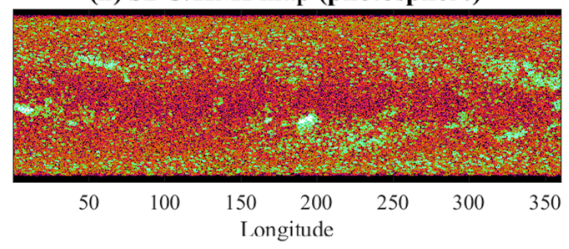

Fig. 6 Magnitude of the vertical electric current density, $\log _{10}\left|j_{r}\right|$, at $r=1.02 R_{\odot}$ in each model, over the whole spherical surface. The color tables are saturated at $10^{-10} \mathrm{G} \mathrm{cm}^{-1}$ (black) and $10^{-6.5} \mathrm{Gcm}^{-1}$ (white). Panel (h) shows the same quantity computed at $r=R_{\odot}$ directly from the SDO/HMI synoptic map in Fig. 2 (boxcar smoothed to reduce noise). The same color scale is used

force-free at the photosphere but becomes rather more so above about $1.1 R_{\odot}$. These differences arise from the physics of the models: the mhd-cese and mhs models include nonmagnetic terms in their force balances, while the ffe model matches to a boundary condition at $r=R_{\odot}$ that does not satisfy $\mathbf{j} \times \mathbf{B}=0$. The model with highest ratio $J / F$ is $m$ hd-mas, owing to the relatively smooth boundary data and level of numerical relaxation applied.

The actual amount of current near the photosphere varies between models, as seen by $J(r)$ in Fig. 6(b). This is highest for $n l f-g r$, because it includes the most fine-scale structure in the HARP patches. On the other hand, $J(r)$ falls off rapidly with height in this model, consistent with the field being closest to potential overall (Table 2 and Fig. $4 b$ ). By contrast, the $m h s$ model has relatively low $J(r)$ near the photosphere, owing to its lower input resolution, and the lowest $J(r)$ is found for the mhd-cese model, which lacks a mechanism for energizing the magnetic field there; its free magnetic energy is located at larger radii where the non-magnetic terms become important.

Both the $m f$ and $m h d$-mas models have lower $J(r)$ very close to the photosphere, because they do not resolve such fine-scale structures as $n l f$ - $g r$ and $n l f$-op. However, these two models have larger current in the low corona $\left(1.1\right.$ to $\left.1.2 R_{\odot}\right)$, arising from the incorporation of low-lying filament channels. These form self-consistently in the $m f$ model, while their locations (for imposed currents) were chosen by design in the mhd-mas model. Between about 1.2 and $1.5 R_{\odot}$, the $m f$ model has the largest $J(r)$, owing to the significant volume currents that have been ejected over time by flux emergence and surface motions. As in the 
nlf-op model, which has almost as high $J(r)$, these currents are not limited to active regions, and reach greater heights in the corona.

Above about $1.7 R_{\odot}$, the ffe model has the largest $J(r)$, although this likely results from incomplete relaxation to equilibrium in the model and deserves further investigation. The $m h d$-cese model also has a more gradual fall-off of $J(r)$ with height than many of the other models, again reflecting the influence of non force-free effects at larger heights in the model as the magnetic field is opened out by the solar wind. Similarly, the $m f$ model becomes non force-free $(J / F<1)$ above about $2 R_{\odot}$, caused by the imposed outflow at the upper boundary. This outflow is used to simulate the effect of the solar wind (Mackay and van Ballegooijen 2006), so that the magnetic field is no longer force-free above $2 R_{\odot}$ but is in a steady-state balance between the outflow and the Lorentz force.

To illustrate the spatial distributions of currents, Fig. 6 shows the magnitude of vertical current density $j_{r}\left(1.02 R_{\odot}, \theta, \phi\right)$ in each model, with the same logarithmic color scale used for all plots. For comparison, panel (h) shows the magnitude of $j_{r}\left(R_{\odot}, \theta, \phi\right)$ computed directly from the SDO/HMI vector synoptic map (Fig. 2).

In all models, the strongest currents are located in the active regions, as might be expected since the magnetic field is strongest there. The magnitudes of these currents differ significantly between the models, consistent with the variations seen in Fig. 5. Outside the active regions, the models differ in their distributions of current. The nlf-op, nlf-gr, $m h s$ and ffe models all show current distributions that correlate with the locations of strongest $\left|j_{r}\right|$ in the photospheric input map (Fig. 6h). These locations are essentially those with strongest large-scale $B_{r}$ in the input map (Fig. 2). Compared to the other models, nlf-op shows a higher background level of current in the quiet Sun, consistent with its values of $J(r)$ and $F(r)$ at this height in Fig. 5. By contrast, $n l f$-gr shows a much lower level of background current outside of active regions, due to the assumption that $j_{r}=0$ in those footpoints. The mhd-cese model has small electric currents at low heights, as previously discussed.

The $m f$ and $m h d$-mas models have additional current concentrations outside the locations of strong observed photospheric $\left|j_{r}\right|$. These take the form of concentrated filament channels, seen in Fig. 6 as parallel lines of $\left|j_{r}\right|$, and lying above polarity inversion lines in the photospheric $B_{r}$. A good example lies between about $60^{\circ}$ and $125^{\circ}$ Carrington longitude in the Southern hemisphere in both models. This current concentration is absent from the other models, and is not seen in the observations at the photospheric level. It is a concentration of electric current density in the coronal volume. We will return to this below in Sect. 3.4.

\subsection{Plane-of-Sky Magnetic Structure}

Figures 7(a) to (g) show visualizations of each model with field lines selected to show the plane-of-sky magnetic structure, as viewed from Earth at approximately the eclipse time. We must bear in mind that, with magnetogram observations presently available only from the Earth's viewpoint, all of the models use primarily synoptic observations built up from central meridian data. These do not include co-temporal information near the limbs, so any comparison can only be approximate. Recall also that the nlf-op and $m h s$ models do not include the region poleward of $\pm 70^{\circ}$ latitude. The model images can be compared to observations of the real corona in EUV (Fig. 7h) and the Fe XIV line (Fig. 7i), as well as in white light out to a larger radius (Fig. 1). These particular observations have been chosen and processed to bring out as clearly as possible the structure of coronal streamers above the limb. References describing the processing are given in the figure caption. When comparing with the models it must be remembered that the observations see total emission along the line-of-sight, which includes structures in front of or behind the sky plane. It is beyond the 
(a) nlf-op

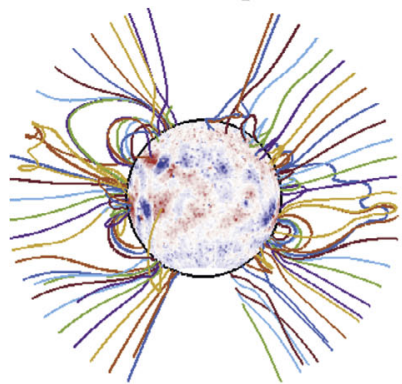

(d) ffe

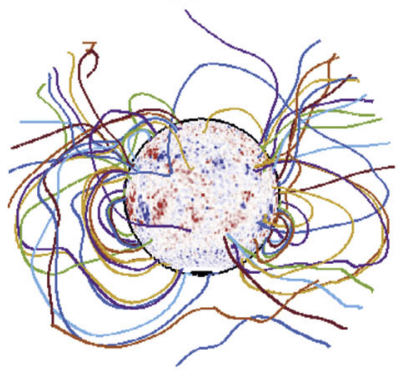

(g) mhd-mas

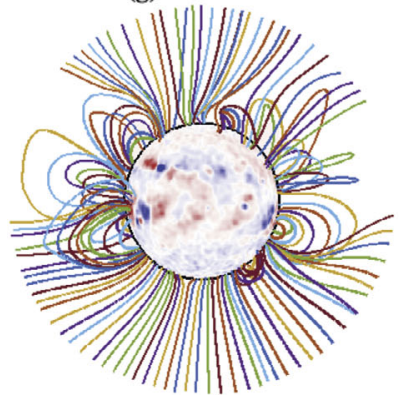

(b) nlf-gr

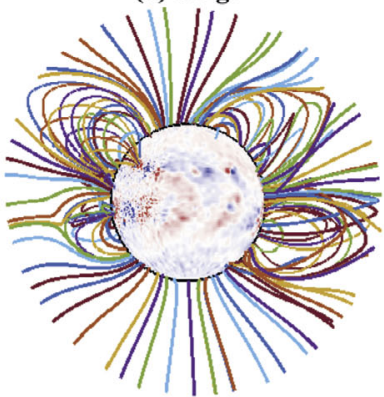

(e) $\mathrm{mf}$

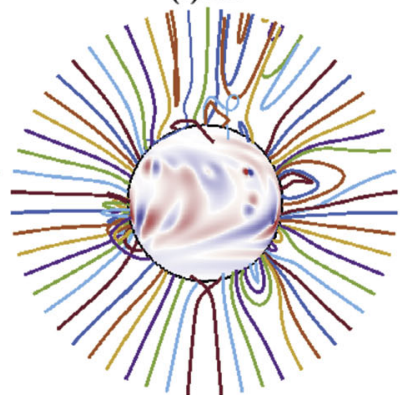

(h) SWAP $174 \mathrm{~A}$

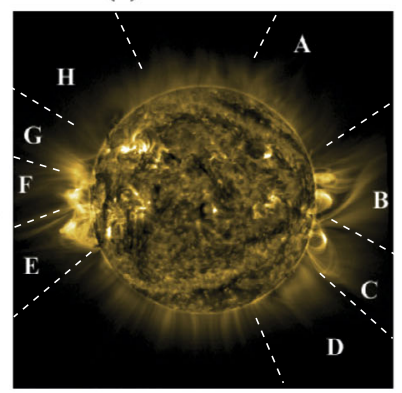

(c) $\mathrm{mhs}$

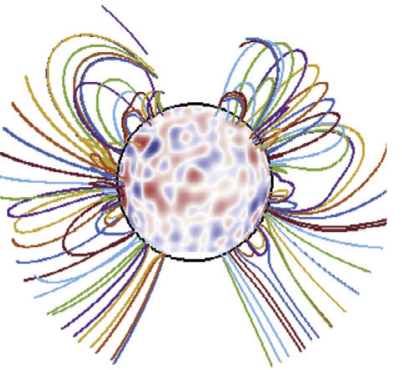

(f) mhd-cese

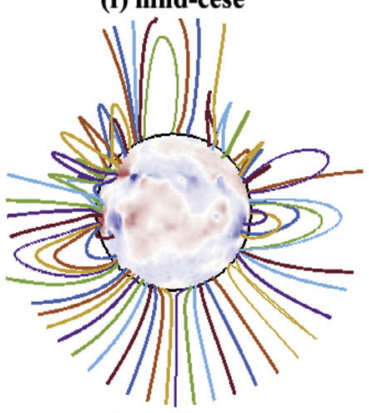

(i) Fe XIV $5303 \mathrm{~A}$

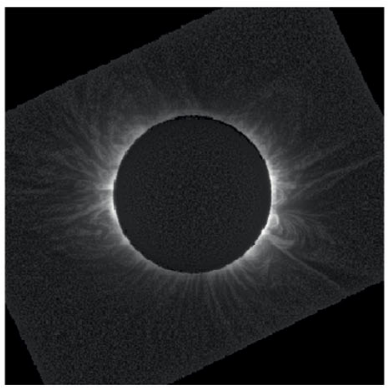

Fig. 7 Magnetic field structure of each model in the plane-of-sky, as viewed from the Earth at the (approximate) eclipse time. (The density of magnetic field lines is arbitrary and does not correspond to field strength.) Red/blue shading shows $B_{r}$ on $r=R_{\odot}$ (saturated at $\pm 50 \mathrm{G}$ ). Panel (h) shows an EUV $174 \AA$ image of the real corona from PROBA2/SWAP (with median stacking and stray-light correction as described by Seaton et al. 2013). Panel (i) shows an image of the corona in the Fe XIV $5305 \AA$ line from the Habbal eclipse expedition, processed by the MGN algorithm of Morgan and Druckmüller (2014) (with parameter $h=0.9$ ) to bring out the streamer structure. Several angular regions are labelled in panel (h) for ease of reference in the text

scope of this project to do forward modelling of coronal emission, particularly since most of the models are purely magnetic. Nevertheless, it is clear that there are significant differences between the streamer structure in the different models, as well as similarities.

Firstly, we observe that streamers (closed field regions) are often too high in many models, particularly mhd-cese, $m h s$, ffe and $n l f$-gr. The white light observations (Fig. 1) suggest their cusps to lie below $2 R_{\odot}$ in most cases, although there could be larger closed loops that cannot be seen due to signal-to-noise issues. In the $n l f$ - $g r$ model, these closed-field regions are close to potential, so the cusp height is set by the potential field source surface at $2.5 R_{\odot}$. 
In the mhd-mas model, the outer boundary condition is one of zero velocity, tending also to keep the streamer cusps at the outer boundary. In ffe and mhd-cese there is no source surface at all, allowing the closed regions to extend even further out. Similarly, the mhs model is an infinite-space solution with no outer boundary imposed. In the $n l f-o p$ and $m f$ models the electric currents allow the streamers to have lower cusps. This is further helped in the $m f$ model by the radial outflow which pulls out the field lines. These differences highlight the importance of the outer boundary conditions for this kind of modelling.

Some models show unusual field line behavior near the outer boundary. For example, $m f$ has disconnected U-loops, which are the result of the ejection of a magnetic flux rope (Mackay and van Ballegooijen 2006; Yeates 2014). Also, some of the field lines for $n l f$-op and ffe are less smooth near the outer boundary. In the case of ffe this is consistent with the large currents seen at larger heights, and the fact that the relaxation has not reached a stable equilibrium. Although these field lines are strongly non-potential, they contribute little to $E$ because the magnetic field strength is weak.

We can also compare the angular positions of particular streamers. These are sensitive to both the input data and the locations of electric currents in the corona. For ease of reference, eight approximate locations are labelled in Fig. 7(h), although these do not necessarily correspond to individual streamers. Looking first at the West limb (A to D), the most prominent streamers in the observations are at B and C. All models show evidence of closed field near these locations, although their morphology differs significantly between models. Except for mhd-cese, the models also tend to agree that there is a narrow pseudo-streamer structure at D. At location A, the observed streamer is less clear in EUV, although there are indications of closed field in Figs. 1 and 7(i). Many of the models show at least some closed field at this location, although its structure and orientation are quite varied between models. As mentioned above, the $m f$ model shows U-loops here resulting from a recent flux rope ejection. Interestingly, there are indications in SWAP of a high-altitude EUV cavity at this location, although it is clearest on the day before that shown in Fig. 7(h). Such a cavity is often associated with an underlying filament or filament channel.

On the East limb, the EUV observations indicate prominent structures at E, F and G around the equator, and there is a filament channel at $\mathrm{H}$ on the polar crown. All models indicate the presence of closed field around E, F and G, associated with the several active regions spread around Carrington longitude $0^{\circ}$. The $m f$ model has rather less closed field here than the other models, owing to the fact that two of the main bipolar regions have not yet reached the visible face of the Sun and been incorporated into the time-evolving $m f$ simulation. This is an example of when additional magnetogram observations around the East limb, such as might be obtained from a satellite at the L5 Lagrange point, would be beneficial if the simulation were to be carried out in real time (Mackay et al. 2016).

At $\mathrm{H}$, all models agree that there is a closed field arcade overlying the East-West polarity inversion line. This is supported by the white light and EUV observations which show a filament at this location. Sheared magnetic field at low heights corresponding to this filament channel has formed naturally in the $m f$ model, and has correspondingly been added to the mhd-mas model. This sheared field was created in $m f$ by differential rotation and flux cancellation at the polarity inversion line, and is not present in the other static models. In these other models, this arcade is closer to potential.

\subsection{Filament Channels}

An important observable signature of non-potential magnetic structure in the corona is the presence of filament channels and filaments. These are located above polarity inversion lines 


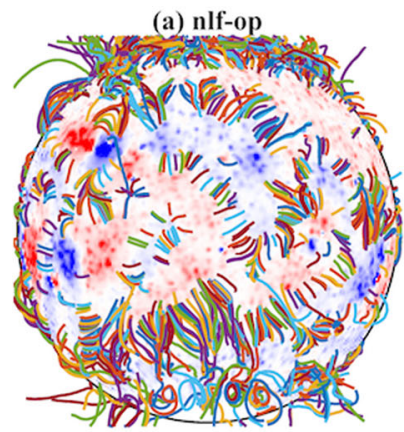

(d) ffe

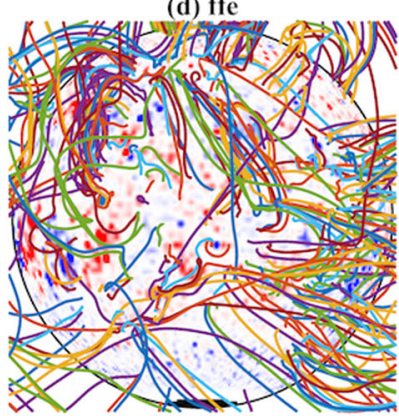

(g) mhd-mas
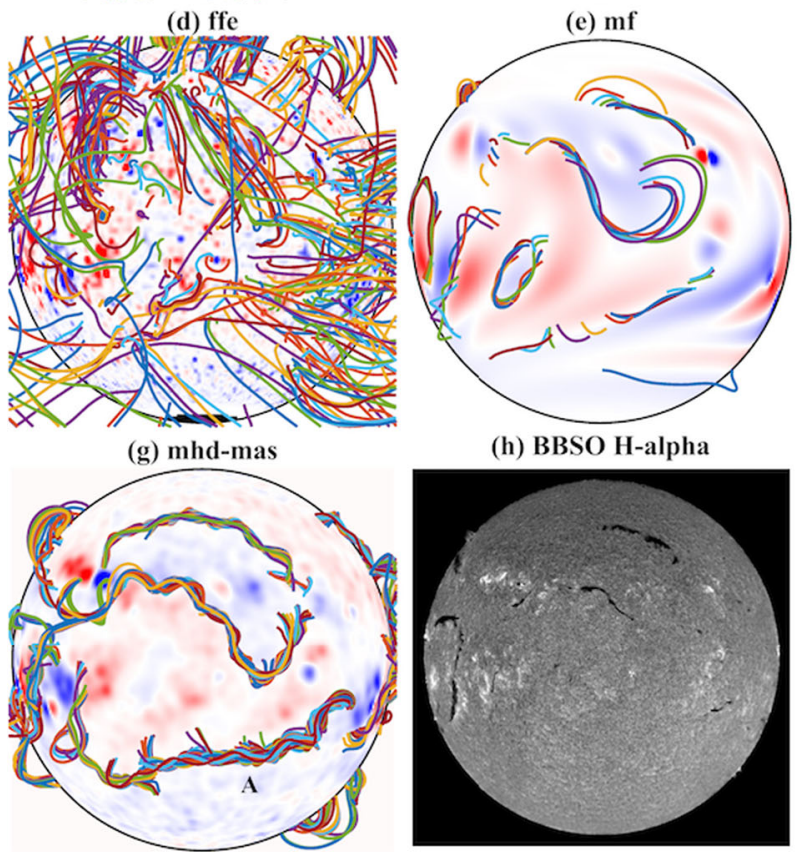

(h) BBSO H-alpha

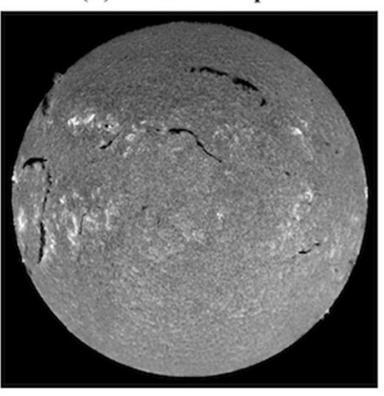

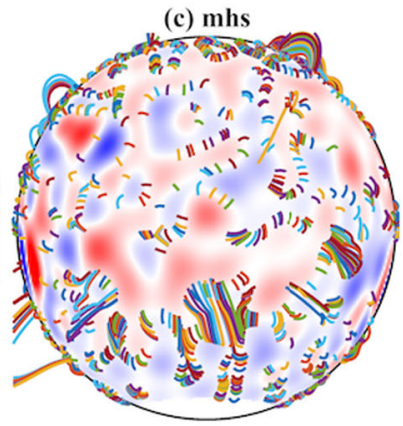

(f) mhd-cese

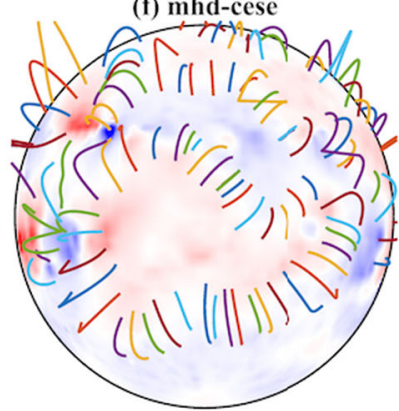

(i) AIA EUV composite

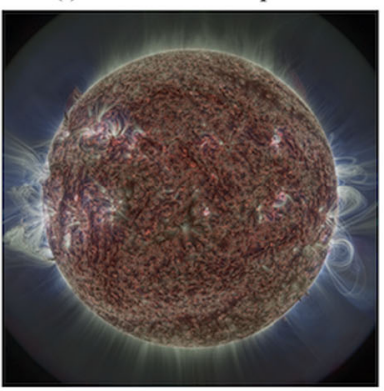

Fig. 8 Magnetic field lines for each model chosen to show the low coronal magnetic structure above each polarity inversion line on the visible disk. Red/blue shading shows $B_{r}$ on $r=R_{\odot}$ (saturated at $\pm 50 \mathrm{G}$ ). Panel (h) shows an $\mathrm{H} \alpha$ image from Big Bear Solar Observatory (taken at 17:33 UT on the eclipse day). Panel (i) shows a composite of the 171, 193 and $211 \AA$ channels (red, green, blue respectively) from AIA, processed as in Fig. 1

in the photospheric $B_{r}$, and are understood to have a strongly sheared magnetic component along the inversion line (Mackay et al. 2010). To illustrate this aspect, Fig. 8 shows a selection of magnetic field lines for each model, this time traced from height $r=1.02 R_{\odot}$ above polarity inversion lines on the solar disk. Observed images in $\mathrm{H} \alpha$ and EUV are shown for comparison.

On the solar disk there are a number of filaments visible in the observations, both in $\mathrm{H} \alpha$ (Fig. 8h) and in EUV (Fig. 8i). One filament extends around the North-East limb as a prominence in the white-light eclipse image (Fig. 1). This is the structure labelled H in Fig. 7(h). The presence of filaments at these particular locations indicates that sheared magnetic field is present. Only the $m f$ and $m h d$-mas models have significantly sheared magnetic field along 
polarity inversion lines. In the case of the $m f$ model, this has built up naturally over time due to surface motions and flux cancellation, whereas in the mhd-mas model it has been imposed based on the $m f$ model results (Appendix A).

In principle, it would be possible for the $n l f-o p$ and $n l f$-gr models to contain sheared fields along these polarity inversion lines. Indeed the $n l f-g r$ model does recover a sigmoidal structure within the active region located around Carrington longitude $190^{\circ}$ (on the far side of the Sun during the eclipse). However, the transverse magnetic field measurements, from which the currents are determined, have a poor signal-to-noise ratio outside of active regions. This arises because the linear polarization scales with $|\mathbf{B}|^{2}$, in contrast to the circular polarization which is linear in $|\mathbf{B}|$. Moreover, by creating synoptic maps, these horizontal magnetic fields and resulting currents are further smeared out. As a result, the corresponding electric currents outside of active regions are not accurate enough to allow the nlf-op and $n l f-g r$ extrapolations to recover the sheared magnetic fields in filament channels. In fact, as we have seen, the $n l f$ - $g r$ model assumed $j_{r}=0$ in the weak field regions of the photosphere, owing to this uncertainty.

The resolution of the BBSO $\mathrm{H} \alpha$ image in Fig. 8 is sufficient to show filaments themselves, as dark structures, but is not sufficient to show empty filament channels. Thus absence of a filament can not be taken to mean absence of sheared magnetic fields. Filament channels may also be identified from alignment of coronal cells in AIA images, particularly in the $193 \AA$ channel (Sheeley and Warren 2012). Careful inspection of SDO/AIA movies of EUV emission, especially using composite images processed by the Morgan and Druckmüller (2014) technique, was used here to identify which filament channels to energize in the mhd-mas model (see Appendix A). This method identifies many filament channels that do not show up clearly in Fig. 8(h) and (i). As a case in point, consider the location labelled A in Fig. 8(g), where a sheared field was inserted in the mhd-mas model. There is only a small amount of $\mathrm{H} \alpha$ filament material visible at the west end of the channel in Fig. 8(h), but analysis of the EUV observations at higher resolution suggests the possible presence of a long East-West filament channel all along this polarity inversion line, bending northward at its eastern end as in the mhd-mas model. A sheared filament channel is also present here in the $m f$ model.

\subsection{Open Magnetic Flux}

The open magnetic field lines are the source of the solar wind, so represent the output of the models as far as the heliosphere is concerned. In Sect. 3.1 (Fig. 4a), we compared the open magnetic flux $\Phi\left(2.5 R_{\odot}\right)$, as defined in Equation (2). The numerical values for each model are given explicitly in Table 3, along with an observational estimate based on in situ OMNI data. To make this estimate, daily averages of the basic hourly OMNI data were obtained from the GSFC/SPDF OMNIWeb interface at http://omniweb.gsfc.nasa.gov, giving $B_{r}\left(R_{\mathrm{E}}\right)$. Averaging over 27 days centered on the eclipse time, and assuming a uniform distribution of magnetic flux over latitude at $R_{\mathrm{E}}=1 \mathrm{AU}$, we estimate the equivalent open flux shown in Table 3 as $\Phi\left(2.5 R_{\odot}\right) \approx 4 \pi R_{\mathrm{E}}^{2}\left|B_{r}\left(R_{\mathrm{E}}\right)\right|=9.05 \times 10^{22} \mathrm{Mx}$. As has previously been found during relatively active periods of solar activity (see Linker et al. 2017), this observed value is much higher than that predicted by models - either potential or non-potential. Our results are consistent with their findings. The additional coronal currents in the $m f$ and $m h d$-cese models, in particular, enhance the open flux significantly, but it still remains below the level inferred from OMNI observations. The reasons for this discrepancy are not yet understood, and are discussed by Linker et al. (2017). It is possible that this may be partially heliospheric in origin, if there are regions in the solar wind where the interplanetary magnetic field folds back on itself and is thus over-counted in the $B_{r}\left(R_{\mathrm{E}}\right)$ measurements (Owens et al. 2013). 
Table 3 Unsigned open magnetic flux $\Phi\left(2.5 R_{\odot}\right)$ of the non-potential models

\begin{tabular}{ll}
\hline Model & $\Phi\left(2.5 R_{\odot}\right)\left[10^{22} \mathrm{Mx}\right]$ \\
\hline nlf-op & 3.27 \\
nlf-gr & 2.67 \\
$m h s$ & 4.37 \\
$f f e$ & 2.69 \\
$m f$ & 5.96 \\
mhd-cese & 5.30 \\
mhd-mas & 2.97 \\
OMNI data (27-day average) & 9.05 \\
\hline
\end{tabular}

Finally, we consider the spatial distribution of open and closed magnetic field in the models. In addition to indicating the possible source regions of the solar wind, this is important because the open or closed nature of the magnetic field at a given location depends sensitively on both the input data and the distribution of electric currents in the model. Comparing the open field footpoints to the locations of observed coronal holes therefore provides a further observational constraint.

Figure 9 shows the locations of open magnetic field line footpoints on $r=R_{\odot}$ for the different models, along with - in panel $(\mathrm{h})$ - a persistence map of observed coronal holes in EUV. This map was built up synoptically over Carrington rotation 2161 (2015 February 28 to 2015 March 27) by thresholding full-disk SDO/AIA $193 \AA$ EUV images, at a cadence of 12 hours. The procedure is described in more detail by Lowder et al. (2017), although during this particular time period no far-side EUV data were available. Of course, this should be taken only as a lower bound on the areas of observed open field rather than a true measure. This is because, while a significant fraction of open flux originates from coronal holes, the footpoints of open field can also be bright, particularly if they lie in active regions. Indeed, quantifying the amount of open flux not located in coronal holes is an important observational challenge for reconciling the models and observations in future.

Given the sensitivity of the open/closed footpoint regions as well as the variety of boundary conditions used, there is reasonable agreement between most of the models and with the observed persistence map. Robust features are labelled in Fig. 9(h) and include: a negativepolarity polar coronal hole in the Southern Hemisphere (A) but no corresponding hole in the Northern hemisphere; a narrow equatorward extension of the Southern polar hole (B and C); a long positive-polarity hole in the Northern hemisphere (D); a north-south oriented positive-polarity hole (E); and a more compact positive-polarity hole near the equator in the Southern Hemisphere $(\mathrm{F})$.

The ffe model differs significantly from the others, as was evident from its magnetic field structure in Fig. 7. The $m f$ model reproduces many of the observed coronal holes, although some (particularly E) are shifted in position. However, it has a number of additional open field regions, with significantly more open magnetic field of both polarities at low and medium latitudes. Indeed this additional open flux was seen in Fig. 4(a). Partly this additional open field arises from the opening up of streamers discussed in Sect. 3.3 and seen in Fig. 7. Another reason for enhancement is the ejection of magnetic flux ropes in the $m f$ model (one such eruption is responsible for the U-loops in Fig. 7e). But the difference from other models is also partly due to the different photospheric distribution of $B_{r}$ (Fig. 3), owing to the model being driven by a flux transport model rather than directly from observed magnetograms (Appendix B). A good example is the large extension of the negative-polarity hole into the Northern hemisphere around Carrington longitude $300^{\circ}$ to $360^{\circ}$ (labelled $\mathrm{G}$ in 


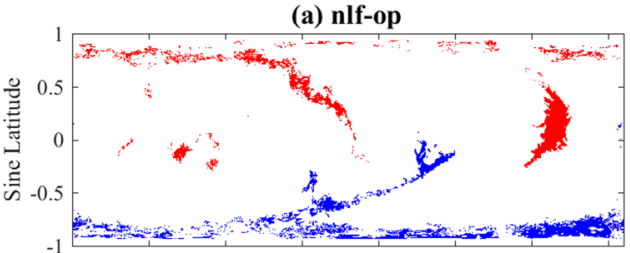

(c) $\mathrm{mhs}$

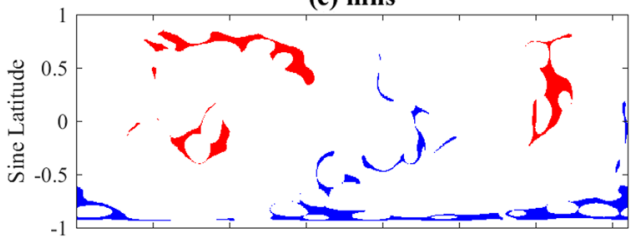

(e) $\mathrm{mf}$

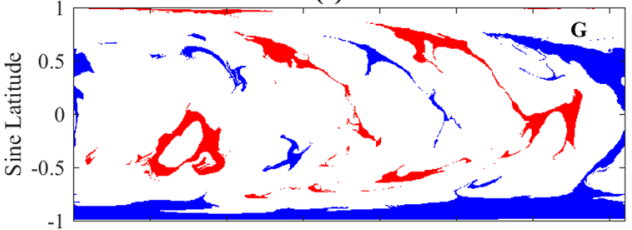

(g) mhd-mas

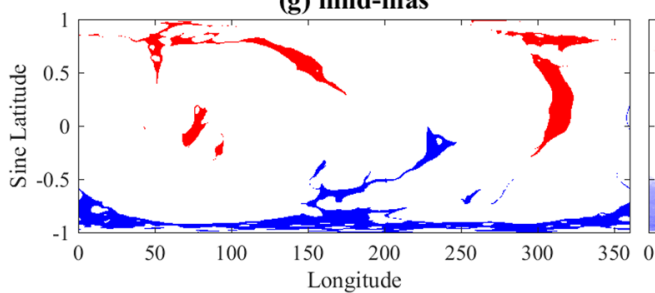

(b) nlf-gr

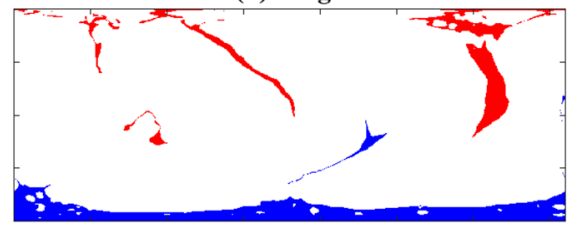

(d) ffe

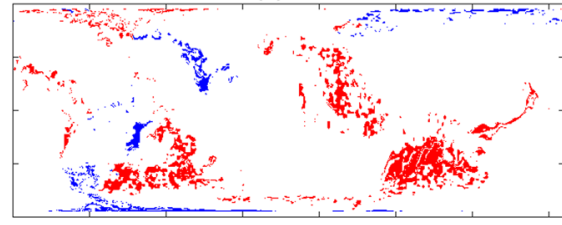

(f) mhd-cese

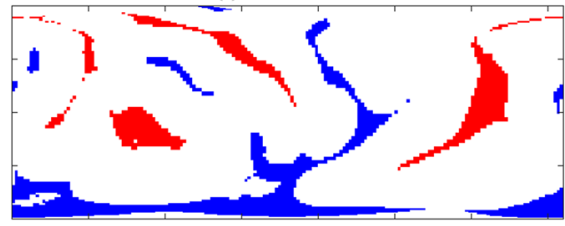

(h) AIA/EUV [persistence]

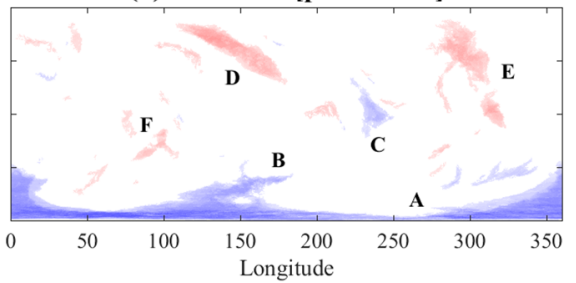

Fig. 9 Footpoints of open magnetic field lines shown on $r=R_{\odot}$, and their polarity (red positive, blue negative). Panel (h) shows the observed coronal hole persistence map (see text) using AIA data. In this map, the colors again indicate polarity, with density indicating persistence, i.e., the number of days for which a coronal hole was observed at that location

Fig. 9e). This hole is likely to reduce in size once the new active regions emerge that are already present in the other models.

Figure 10 shows the distribution of $B_{r}$ on the outer boundary $r=2.5 R_{\odot}$ in each model. Here all of the field lines are "open" according to our definition. At this radius, the magnetic field is insensitive to small-scale differences in the input magnetic maps, and there is reasonable agreement between $n l f$-op, $n l f$-gr, $m h s$ and $m h d$-mas. These models all inherit the same basic open/closed topology from the potential field used as their initial condition before the injection of electric currents at the base. In the ffe, $m f$ and mhd-cese models, the heliospheric current sheet (boundary between positive and negative $B_{r}$ ) has a more complex shape, with a disconnected loop in the $m f$ model. The reasons for these differences are the same as in Fig. 9. As mentioned above, the ffe model has a complex magnetic structure that likely reflects the incomplete relaxation of the model. These results do suggest that we should not take the potential field topology for granted, although some of these differences arise from the different photospheric $B_{r}$ distribution in the $m f$ and $m h d$-cese models; indeed, the coronal hole map comparison suggests that the difference may be over-emphasized in these models. The additional open flux in the $m h s, m h s$ and $m h d$-cese models, compared to 

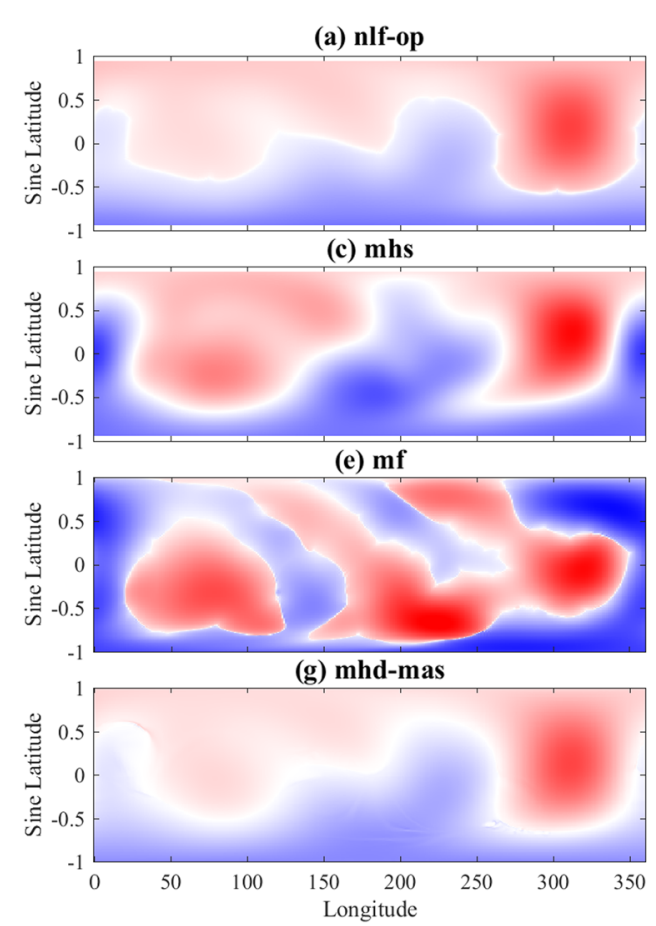

(b) nlf-gr

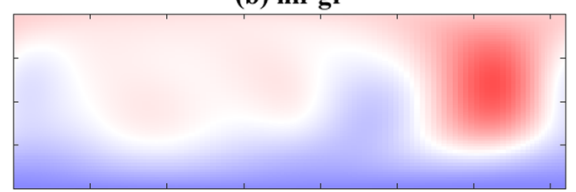

(d) ffe

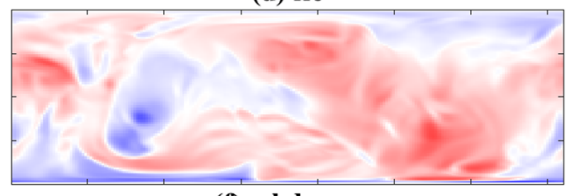

(f) mhd-cese

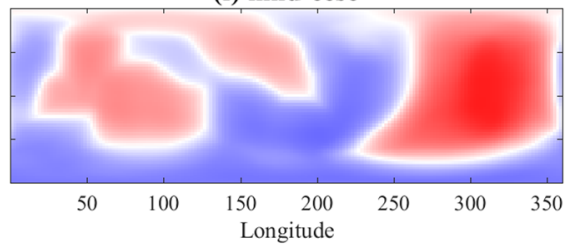

Fig. 10 Radial magnetic field at $r=2.5 R_{\odot}$ in each model, over the full spherical surface. The color tables are saturated at $\pm 0.4 \mathrm{G}$

the others, is also clear in this plot. Note that this could be reduced in the $m h s$ model by reducing the parameter $a$, so may not be particularly significant in that case.

\section{Conclusions}

Having analysed seven different non-potential models of the coronal magnetic field on 2015 March 20, we now draw some overall conclusions.

The initial impression from Fig. 7, for example, is one of significant disagreement between different models. This is true particularly in regard to the overall magnetic structure. This disagreement arises from several sources: the input data used by each modeller, the coronal modelling techniques themselves, and the outer boundary conditions.

There are two fundamental limitations with currently available magnetogram input, not including the differences between magnetograms from different instruments and observatories (Riley et al. 2014), which we avoid here by focusing on SDO/HMI. One limitation is the lack of co-temporal full surface coverage, which necessitates the use of synoptic maps that combine observations taken at different times. The different models achieve this in different ways, as described in Sect. 2, and this leads to different maps of the magnetic field across the whole solar surface (Fig. 3), with corresponding differences in coronal magnetic topology. One consequence of the use of synoptic maps is that the majority of models (except for $m f$ and $m h d$-cese) use data taken after the eclipse time, meaning that this exercise is not equivalent to a prediction of the eclipse magnetic field which could have been made in 
advance. The $B_{r}$ distribution in the $m f$ model differs particularly from the others because it uses a surface flux transport model to evolve the photospheric magnetic field over a period of months, rather than inserting magnetogram data more directly. This has the advantage of allowing the build-up of free magnetic energy in weak-field regions, but does mean that there are resulting differences in the magnetic structure. The use of synoptic data also means that the magnetogram input near the limbs - particularly the East limb - is out-of-date, and this can particularly affect comparisons with eclipse images. The proposed space mission to the L5 Lagrange point would greatly improve the situation at the East limb, provided that a magnetograph were included on board.

The second limitation is with vector magnetograms. Firstly, the signal-to-noise ratio remains low in the transverse components of $\mathbf{B}$, so that the $n l f-o p, n l f-g r$ and ffe models do not reproduce the sheared magnetic fields in filament channels. And the fact that the photospheric vector magnetograms do not satisfy $\mathbf{j} \times \mathbf{B}=0$ leads to problems in particular with the ffe model, preventing it from reaching equilibrium. Further refinement will be needed before this model can be of practical use for the solar corona. In future, it is hoped that this issue will improve if and when upper-chromospheric magnetogram observations become available.

In the coronal volume itself, we have shown that the different models vary in their degree of non-potentiality, as measured by either electric currents or free energy. The models with greater free energy achieve this with a variety of different current distributions: currents may be concentrated in active regions ( $n l f$-gr), in filament channels ( $m h d$-mas and $m f$ ), or may be more distributed throughout the corona ( $m f$ and $m h s$ ). This aspect of the models is perhaps the most difficult to calibrate against observations, since no direct observations of coronal electric currents are available. The presence of filament channels, for example, illustrates the importance of the gradual build-up of coronal electric currents over time. Although these are not reproduced in the $n l f$-gr, $n l f-o p, m h s$ and $m h d$-cese models, this is not due to a fundamental limitation of the equations used to model the magnetic field in the corona, but due to limitations in the available boundary data. In particular, we have shown with the $m h d$ mas model how static models can be further energized by the injection of electric currents in filament channels, and this could be applied in future with the other models. This hybrid approach of informing static models with the results either from simplified time-evolving models (like $m f$ ) or from additional coronal observations could well be a useful one in the absence of improved magnetogram input data.

The outer boundary conditions also have a significant impact, and deserve greater attention. With the exception of $m h d$-cese, none of the models presented here couples physically to a solar wind solution beyond $2.5 R_{\odot}$. This is because the increasing plasma- $\beta$ in that region no longer allows for the use of a purely magnetic model. Several of the models impose an artificial source surface at $2.5 R_{\odot}$, as in the common PFSS model, and this leads to inaccuracies in the streamer structure, and potentially also the open flux. The $m f$ model instead uses a radial outflow boundary condition to mimic the effect of the solar wind, but this seems to be inflating the field too much in this particular case, at least in terms of open field footpoints (though interestingly not in the height of the streamers). The corresponding currents higher in the corona change the open-closed magnetic topology significantly. Overall, this outer boundary is an important problem that requires more sophisticated MHD modelling that includes plasma thermodynamics. Such simulations have been performed with the fullMHD version of the mhd-mas model (e.g., Lionello et al. 2009; Downs et al. 2013), which can describe the solar wind.

All of this being said, there are also areas of broad agreement between many of the models. For example, while they have very different input grid resolutions on $r=R_{\odot}$, this 
(in itself) does not affect the estimated open flux and topology of the heliospheric current sheet on $r=2.5 R_{\odot}$, which arises rather from the differences between the coronal modelling approaches. On $r=R_{\odot}$, the footpoint regions of open magnetic field show similarities in all models, and there is agreement that the strongest currents are within the active regions. Among those models with significant free magnetic energy, there is general agreement on the ratio $E / E_{\mathrm{p}} \approx 1.4$ to 1.5 . And the locations of closed field streamers are broadly in agreement, though not their height and shape.

From this study it is clear that all of the models have positive aspects that agree with observations, but other aspects that do not match so well. Much of this can be related to the distribution of electric current both within and outside of active regions. At present, nonlinear force-free extrapolations such as $n l f$-op or $n l f$ - $g r$ are best at representing the structure of active regions where reliable vector magnetic field input data are available. But accounting for the free energy outside of active regions is currently possible only with time-evolving models such as $m f$. Yet, in these models, it is too computationally expensive to account for the full plasma thermodynamics, something that has not been considered here but is already possible in state-of-the-art full-MHD models, albeit for static configurations.

What is clear is that this is an exciting time for coronal magnetic field modelling, with progress on several fronts but much still to do. Rather than simply waiting for better magnetogram data, our comparisons with currently available observations - though qualitative - do suggest that these indirect observational constraints could be better used to optimize the models. The challenge is to do this systematically. The ideal model would match EUV observations of filament channels and coronal loops, the positions of white-light streamers, and the locations of observed coronal holes. A more sophisticated approach would involve forward modelling of actual observed emission, but we suggest that much can already be learned from morphological comparisons.

Acknowledgements The authors gratefully thank ISSI Bern for hosting our International Team and enabling this study to be carried out. The $n l f$-gr work was granted access to the HPC resources of CINES/IDRIS under the allocation 2016-16050438 made by GENCI. ARY and DHM thank the UK STFC for financial support. ARY and CAL were supported by a grant from the US Air Force Office for Scientific Research. DHM also thanks the Leverhulme Trust for financial support. GP acknowledges support from NASA grant NNX15AN43G. FXS was supported by the National Natural Science Foundation of China (Grant No. 41531073). TW acknowledges DFG-grant WI 3211/5-1. Authors from Predictive Science, Inc. were supported by NASA, NSF, and AFOSR, including support from NASA grant NNX15AB65G. Their computational resources were provided by NASA's Advanced Supercomputing Division and NSF's XSEDE program. LAU was supported by the National Science Foundation Atmospheric and Geospace Sciences Postdoctoral Research Fellowship Program. National Center for Atmospheric Research is sponsored by the National Science Foundation. HM's research is partly funded by the Leverhulme Foundation and an STFC PR\&D grant. The eclipse data is provided through the kind permission of Prof Shadia Habbal at the University of Hawaii who leads an international team of eclipse scientists.

Open Access This article is distributed under the terms of the Creative Commons Attribution 4.0 International License (http://creativecommons.org/licenses/by/4.0/), which permits unrestricted use, distribution, and reproduction in any medium, provided you give appropriate credit to the original author(s) and the source, provide a link to the Creative Commons license, and indicate if changes were made.

\section{Appendix A: Details of the mhd-mas Model}

The MAS time-dependent magnetohydrodynamic (MHD) model (Mikic and Linker 1994; Linker and Mikic 1995; Mikić et al. 1999) of the global solar corona has been in development for over two decades. The simulation mhd-mas illustrated in this paper uses a simplified zero-beta version of the model, in which pressure and gravity forces are neglected, in 
order to emphasize the non-potential aspects of the corona, in particular the sheared fields expected in filament channels. An energization mechanism was applied to create sheared and twisted magnetic fields and flux ropes in the observed filament channels. Unlike the more sophisticated version of the MHD model, this zero-beta version is not able to include explicitly the effect of the solar wind, but in the future it is hoped to include this energization capability into the full MHD model.

The filaments (visible for example in Fig. 8h) are thought to be supported by twisted flux-rope-like magnetic fields. These were introduced in an ad hoc manner as follows:

1. Start with a PFSS solution but add additional flux to $B_{r}$ on the boundary $r=R_{\odot}$, to compensate for that lost by the cancellation in Step 3.

2. Emerge transverse magnetic field by imposing a transverse electric field $\mathbf{E}_{t}=\nabla_{t} \Phi$ at the boundary $r=R_{\odot}$. Here $\Phi$ is chosen to be localized to the filament channels, and reverses sign across the PILs, and $\nabla_{t}$ is the transverse gradient. Note that this does not change $B_{r}$.

3. After the transverse magnetic field emergence is complete, cancel flux at the PILs by applying a transverse electric field of the form $\mathbf{E}_{t}=\nabla_{t} \times \psi \hat{\mathbf{r}}$, so as to form more fluxrope like field lines out of highly sheared arcades. The function $\psi$ is driven by the change in $B_{r}$,

$$
\frac{1}{c} \frac{\partial B_{r}}{\partial t}=\nabla_{t}^{2} \psi
$$

By the end of this process, $B_{r}$ will once again match the magnetogram, thanks to the additional flux added in Step 1.

The total amount of added flux, which was chosen by trial and error, corresponds to $20 \%$ of the flux in the magnetogram (over the whole Sun). Since the added flux is localized to filament channels, it corresponds to a greater fraction of the flux in the filament channels (on the order of $40 \%$ ).

The filament channel regions at which the field was twisted in this manner were chosen in an ad hoc manner, guided by both the results of the $m f$ model and the locations of filament channels inferred from SDO/AIA EUV movies during the evolution surrounding the eclipse. Since the $m f$ model assimilated the emerging active regions from HMI observations over time, it was able to give the best estimate of the chirality of the fields in the filament channels. By chirality here we mean the direction of the transverse field introduced. According to the usual definition, a sinistral filament has an axial field that points to the left when looking towards the PIL from the side with $B_{r}>0$, and vice versa for a dextral filament. In the technique described above, choosing $\Phi$ with the same sign as $B_{r}$ produces a dextral filament, whereas $\Phi$ with opposite sign to $B_{r}$ produces a sinistral filament. In some cases, when the chirality changed within a single filament, a single chirality was used to simplify matters. Figure 11 shows the filament channels where the magnetic fields were energized, with the chosen chirality (as deduced from the $m f$ model), and the corresponding $\Phi$ potential used. The magnetic energy of the initial PFSS field (with the observed $B_{r}$ plus the added flux) was $2.13 \times 10^{33} \mathrm{ergs}$. The energy in the PFSS field corresponding to the observed $B_{r}$ was $1.72 \times 10^{33}$ ergs. The energy in the final field, with twisted fields in the filament channels, matching the observed $B_{r}$, was $2.52 \times 10^{33}$ ergs, so that the final field had an energy $47 \%$ above the corresponding PFSS field.

\section{Appendix B: Details of the $m f$ Model}

For the $m f$ calculation presented here, the coronal magnetic field was evolved for 200 days up to the eclipse date, allowing the self consistent build-up of electric currents and free 


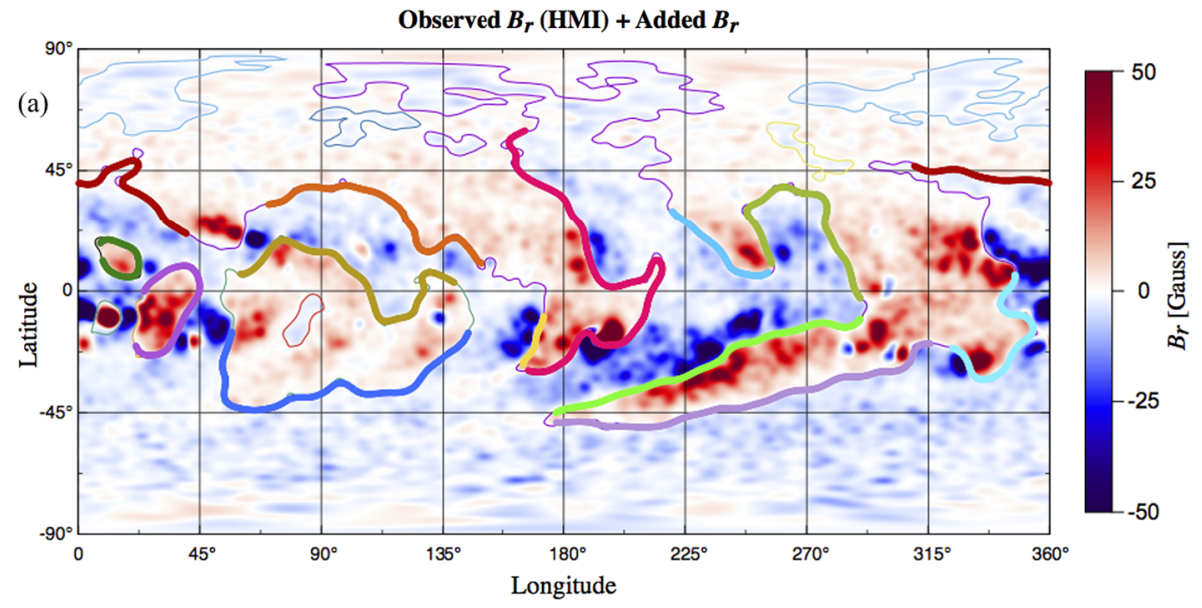

$\Phi$ Potential, Energized Filament Channels, and Chirality

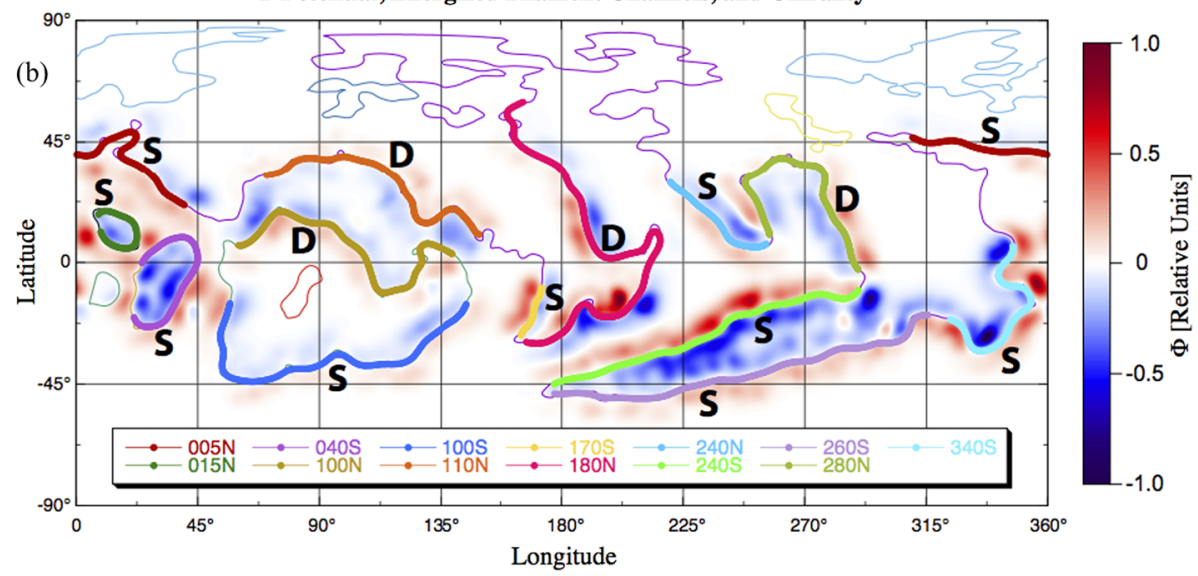

Fig. 11 Energization of filament channels in the mhd-mas model. Panel (a) shows radial magnetic field $B_{r}$ at $r=R_{\odot}$ for the observed HMI field with the added flux. The colored contours are the PILs in the PFSS model at $r=1.02 R_{\odot}$, with thick colored line segments showing the filament channels in which the field was energized. Panel (b) shows the energizing $\Phi$ potential. The chirality selected for the magnetic field in these filament channels is shown in (b), with " $\mathrm{S}$ " denoting sinistral and " $\mathrm{D}$ " denoting dextral filaments

magnetic energy, driven by the photospheric evolution (both footpoint motions and flux emergence). The simulation was initialized with a potential field extrapolation for 2014 September 1, and a fully non-potential corona was arrived at after 6-8 weeks (as measured by average current density), well before the eclipse date.

Compared to previously published simulations, a different evolution of $B_{r}$ at the solar surface $r=R_{\odot}$ was used. Namely, this was derived from the Advective Flux Transport (AFT) model of Upton and Hathaway (2014b,a) and Ugarte-Urra et al. (2015), which assimilates HMI magnetograms on the visible disk and advects the distribution over the full solar surface using differential rotation, meridional flow and small-scale convective flows. For driving the magneto-frictional model, it is actually the horizontal electric field that is required. Since this is unknown in the regions assimilated from HMI, the AFT model could not be used directly to drive the $3 \mathrm{D}$ simulations. Instead, it was used to de- 
termine the locations of new bipolar magnetic regions (BMRs), which were then inserted into a simpler flux transport model which also includes differential rotation and meridional circulation, but approximates the small-scale convective flows by a simple supergranular diffusion term. The resulting smoother distribution of the photospheric field is evident in Fig. 3(e).

The BMR properties were derived from the AFT model by a three-stage automated procedure:

1. Compare successive $B_{r}$ maps (once every $24 \mathrm{hrs)}$ to identify new BMRs (leading to 197 distinct BMRs).

2. Compute the BMR properties (location, size, magnetic flux and tilt angle) once per hour, from $48 \mathrm{hrs}$ before to $120 \mathrm{hrs}$ after initial identification, and determine the time of maximum flux. Insert the (3D) BMR in the new simulation at this time with the corresponding properties.

3. Check the resulting $B_{r}$ evolution manually, and correct the BMR properties to best reproduce their times of emergence and also the structure of multiple-BMR activity complexes.

In the run shown here, each BMR was given a twist when inserted into the $3 \mathrm{D}$ simulation so as to model the active region helicity - with magnitude $\beta= \pm 0.4$ (see Yeates et al. 2008).

\section{References}

T. Amari, J.-J. Aly, A. Canou, Z. Mikic, Reconstruction of the solar coronal magnetic field in spherical geometry. Astron. Astrophys. 553, A43 (2013). https://doi.org/10.1051/0004-6361/201220787

T. Amari, J.-J. Aly, P. Chopin, A. Canou, Z. Mikic, Large scale reconstruction of the solar coronal magnetic field. J. Phys. Conf. Ser. 544, 012012 (2014). https://doi.org/10.1088/1742-6596/544/1/012012

M.G. Bobra, X. Sun, J.T. Hoeksema, M. Turmon, Y. Liu, K. Hayashi, G. Barnes, K.D. Leka, The Helioseismic and Magnetic Imager (HMI) vector magnetic field pipeline: SHARPs - Space-Weather HMI Active Region Patches. Sol. Phys. 289, 3549 (2014). https://doi.org/10.1007/s11207-014-0529-3

T.J. Bogdan, B.C. Low, The three-dimensional structure of magnetostatic atmospheres, II: modeling the largescale corona. Astrophys. J. 306, 271 (1986). https://doi.org/10.1086/164341

I. Contopoulos, The force-free electrodynamics method for the extrapolation of coronal magnetic fields from vector magnetograms. Sol. Phys. 282, 419 (2013). https://doi.org/10.1007/s11207-012-0154-y

I. Contopoulos, C. Kalapotharakos, M.K. Georgoulis, Nonlinear force-free reconstruction of the global solar magnetic field: methodology. Sol. Phys. 269, 351 (2011). https://doi.org/10.1007/s11207-011-9713-X

M.L. DeRosa, M.S. Wheatland, K.D. Leka, G. Barnes, T. Amari, A. Canou, S.A. Gilchrist, J.K. Thalmann, G. Valori, T. Wiegelmann, C.J. Schrijver, A. Malanushenko, X. Sun, S. Régnier, The influence of spatial resolution on nonlinear force-free modeling. Astrophys. J. 811, 107 (2015). https://doi.org/10.1088/ 0004-637X/811/2/107

C. Downs, J.A. Linker, Z. Mikić, P. Riley, C.J. Schrijver, P. Saint-Hilaire, Probing the solar magnetic field with a Sun-Grazing comet. Science 340, 1196 (2013). https://doi.org/10.1126/science. 1236550

M. Druckmüller, Phase correlation method for the alignment of total solar eclipse images. Astrophys. J. 706, 1605 (2009). https://doi.org/10.1088/0004-637X/706/2/1605

X. Feng, L. Yang, C. Xiang, C. Jiang, X. Ma, S.T. Wu, D. Zhong, Y. Zhou, Validation of the 3D AMR SIP-CESE solar wind model for four carrington rotations. Sol. Phys. 279, 207 (2012). https://doi.org/ 10.1007/s11207-012-9969-9

J.T. Hoeksema, Y. Liu, K. Hayashi, X. Sun, J. Schou, S. Couvidat, A. Norton, M. Bobra, R. Centeno, K.D. Leka, G. Barnes, M. Turmon, The Helioseismic and Magnetic Imager (HMI) vector magnetic field pipeline: overview and performance. Sol. Phys. 289, 3483 (2014). https://doi.org/10.1007/ s11207-014-0516-8

J.A. Linker, Z. Mikic, Disruption of a helmet streamer by photospheric shear. Astrophys. J. Lett. 438, L45 (1995). https://doi.org/10.1086/187711

J.A. Linker, R.M. Caplan, C. Downs, P. Riley, Z. Mikic, R. Lionello, C.J. Henney, C.N. Arge, Y. Liu, M.L. Derosa, A. Yeates, M.J. Owens, The open flux problem. Astrophys. J. 848, 70 (2017). https://doi.org/ $10.3847 / 1538-4357 / \mathrm{aa} 8 \mathrm{a} 70$ 
R. Lionello, J.A. Linker, Z. Mikić, Multispectral emission of the Sun during the first whole sun month: magnetohydrodynamic simulations. Astrophys. J. 690, 902 (2009). https://doi.org/10.1088/ 0004-637X/690/1/902

C. Lowder, J. Qiu, R. Leamon, coronal holes and open magnetic flux over cycles 23 and 24. Sol. Phys. 292, 18 (2017). https://doi.org/10.1007/s11207-016-1041-8

D.H. Mackay, A.A. van Ballegooijen, Models of the large-scale corona, I: formation, evolution, and liftoff of magnetic flux ropes. Astrophys. J. 641, 577 (2006). https://doi.org/10.1086/500425

D.H. Mackay, A.R. Yeates, The Sun's global photospheric and coronal magnetic fields: observations and models. Living Rev. Sol. Phys. 9, 6 (2012). https://doi.org/10.12942/lrsp-2012-6

D.H. Mackay, A.R. Yeates, F.-X. Bocquet, Impact of an L5 magnetograph on nonpotential solar global magnetic field modeling. Astrophys. J. 825, 131 (2016). https://doi.org/10.3847/0004-637X/825/2/131

D.H. Mackay, J.T. Karpen, J.L. Ballester, B. Schmieder, G. Aulanier, Physics of solar prominences, II: magnetic structure and dynamics. Space Sci. Rev. 151, 333 (2010). https://doi.org/10.1007/ s11214-010-9628-0

Z. Mikic, J.A. Linker, Disruption of coronal magnetic field arcades. Astrophys. J. 430, 898 (1994). https:// doi.org/10.1086/174460

Z. Mikić, J.A. Linker, D.D. Schnack, R. Lionello, A. Tarditi, Magnetohydrodynamic modeling of the global solar corona. Phys. Plasmas 6, 2217 (1999). https://doi.org/10.1063/1.873474

H. Morgan, M. Druckmüller, Multi-scale Gaussian normalization for solar image processing. Sol. Phys. 289, 2945 (2014). https://doi.org/10.1007/s11207-014-0523-9

M.J. Owens, N.U. Crooker, M. Lockwood, Solar origin of heliospheric magnetic field inversions: evidence for coronal loop opening within pseudostreamers. J. Geophys. Res. Space Phys. 118, 1868 (2013). https:// doi.org/10.1002/jgra.50259

G. Petrie, High-resolution vector magnetograms of the sun's poles from hinode: flux distributions and global coronal modeling. Sol. Phys. 292, 13 (2017). https://doi.org/10.1007/s11207-016-1034-7

P. Riley, M. Ben-Nun, J.A. Linker, Z. Mikic, L. Svalgaard, J. Harvey, L. Bertello, T. Hoeksema, Y. Liu, R. Ulrich, A multi-observatory inter-comparison of line-of-sight synoptic solar magnetograms. Sol. Phys. 289, 769 (2014). https://doi.org/10.1007/s11207-013-0353-1

J. Schou, P.H. Scherrer, R.I. Bush, R. Wachter, S. Couvidat, M.C. Rabello-Soares, R.S. Bogart, J.T. Hoeksema, Y. Liu, T.L. Duvall, D.J. Akin, B.A. Allard, J.W. Miles, R. Rairden, R.A. Shine, T.D. Tarbell, A.M. Title, C.J. Wolfson, D.F. Elmore, A.A. Norton, S. Tomczyk, Design and ground calibration of the Helioseismic and Magnetic Imager (HMI) instrument on the Solar Dynamics Observatory (SDO). Sol. Phys. 275, 229 (2012). https://doi.org/10.1007/s11207-011-9842-2

C.J. Schrijver, M.L. DeRosa, T. Metcalf, G. Barnes, B. Lites, T. Tarbell, J. McTiernan, G. Valori, T. Wiegelmann, M.S. Wheatland, T. Amari, G. Aulanier, P. Démoulin, M. Fuhrmann, K. Kusano, S. Régnier, J.K. Thalmann, Nonlinear force-free field modeling of a solar active region around the time of a major flare and coronal mass ejection. Astrophys. J. 675, 1637 (2008). https://doi.org/10.1086/527413

D.B. Seaton, A. De Groof, P. Shearer, D. Berghmans, B. Nicula, SWAP observations of the long-term, large-scale evolution of the extreme-ultraviolet solar corona. Astrophys. J. 777, 72 (2013). https:// doi.org/10.1088/0004-637X/777/1/72

N.R. Sheeley, H.P. Warren, Coronal cells. Astrophys. J. 749, 40 (2012). https://doi.org/10.1088/ 0004-637X/749/1/40

T. Tadesse, T. Wiegelmann, B. Inhester, Nonlinear force-free coronal magnetic field modelling and preprocessing of vector magnetograms in spherical geometry. Astron. Astrophys. 508, 421 (2009). https://doi.org/10.1051/0004-6361/200912529

T. Tadesse, T. Wiegelmann, S. Gosain, P. MacNeice, A.A. Pevtsov, First use of synoptic vector magnetograms for global nonlinear, force-free coronal magnetic field models. Astron. Astrophys. 562, A105 (2014). https://doi.org/10.1051/0004-6361/201322418

I. Ugarte-Urra, L. Upton, H.P. Warren, D.H. Hathaway, Magnetic flux transport and the long-term evolution of solar active regions. Astrophys. J. 815, 90 (2015). https://doi.org/10.1088/0004-637X/815/2/90

L. Upton, D.H. Hathaway, Effects of meridional flow variations on solar cycles 23 and 24. Astrophys. J. 792, 142 (2014a). https://doi.org/10.1088/0004-637X/792/2/142

L. Upton, D.H. Hathaway, Predicting the sun's polar magnetic fields with a surface flux transport model. Astrophys. J. 780, 5 (2014b). https://doi.org/10.1088/0004-637X/780/1/5

T. Wiegelmann, Computing nonlinear force-free coronal magnetic fields in spherical geometry. Sol. Phys. 240, 227 (2007). https://doi.org/10.1007/s11207-006-0266-3

T. Wiegelmann, T. Sakurai, Solar force-free magnetic fields. Living Rev. Sol. Phys. 9, 5 (2012). https:// doi.org/10.12942/lrsp-2012-5 
T. Wiegelmann, G.J.D. Petrie, P. Riley, Coronal magnetic field models. Space Sci. Rev. 210, 249 (2017). https://doi.org/10.1007/s11214-015-0178-3

A.R. Yeates, Coronal magnetic field evolution from 1996 to 2012: continuous non-potential simulations. Sol. Phys. 289, 631 (2014). https://doi.org/10.1007/s11207-013-0301-0

A.R. Yeates, D.H. Mackay, A.A. van Ballegooijen, Modelling the global solar corona, II: coronal evolution and filament chirality comparison. Sol. Phys. 247, 103 (2008). https://doi.org/10.1007/ s1 1207-007-9097-0 